Marquette University

e-Publications@Marquette

Mechanical Engineering Faculty Research and

Publications

Mechanical Engineering, Department of

4-2019

\title{
Geometry Based Synthesis of Planar Compliances with Redundant Mechanisms Having Five Compliant Components
}

Shuguang Huang

Marquette University, shuguang.huang@marquette.edu

Joseph M. Schimmels

Marquette University, joseph.schimmels@marquette.edu

Follow this and additional works at: https://epublications.marquette.edu/mechengin_fac

Part of the Mechanical Engineering Commons

\section{Recommended Citation}

Huang, Shuguang and Schimmels, Joseph M., "Geometry Based Synthesis of Planar Compliances with Redundant Mechanisms Having Five Compliant Components" (2019). Mechanical Engineering Faculty Research and Publications. 242.

https://epublications.marquette.edu/mechengin_fac/242 
Marquette University

e-Publications@Marquette

\title{
Mechanical Engineering Faculty Research and Publications/College of Engineering
}

This paper is NOT THE PUBLISHED VERSION; but the author's final, peer-reviewed manuscript. The published version may be accessed by following the link in th citation below.

Mechanism and Machine Theory, Vol. 134 (April 2019): 645-666. DOI. This article is (C) Elsevier and permission has been granted for this version to appear in e-Publications@Marquette. Elsevier does not grant permission for this article to be further copied/distributed or hosted elsewhere without the express permission from Elsevier.

\section{Geometry Based Synthesis of Planar Compliances with Redundant Mechanisms Having Five Compliant Components}

\author{
Shuguang Huang \\ Department of Mechanical Engineering, Marquette University, Milwaukee, WI \\ Joseph M. Schimmels \\ Department of Mechanical Engineering, Marquette University, Milwaukee, WI
}

\begin{abstract}
In this paper, a geometric approach to the passive realization of any planar compliance with a redundant compliant mechanism is presented. The mechanisms considered are either simple serial mechanisms consisting of five elastic joints or simple parallel mechanisms consisting of five springs. For each type of mechanism, realization conditions to achieve a given compliance are derived. The physical significance of each condition is identified and graphically interpreted. Geometry based synthesis procedures to achieve any given compliance are developed for both types of mechanisms. Since each realization condition imposes restrictions solely on the
\end{abstract}


mechanism geometry, the procedures allow one to choose the geometric properties of each component (from a set of admissible options) independently from the selection of the elastic properties of each component.

\section{Keywords}

Compliance synthesis, Passive realization of compliance, Redundant compliant mechanisms

\section{Introduction}

In robotic manipulation, compliance is a common means of providing force regulation and stable positioning relative to constraints [1], [2]. For small, quasi-static elastic deformations from equilibrium, a linear relationship exists between a twist (the displacement and rotation in Cartesian space) and a wrench (force and moment in Cartesian space). This relationship can be represented by a symmetric positive semidefinite (PSD) matrix, the compliance matrix $\mathbf{C}$, or the stiffness matrix $\mathbf{K}$, the inverse of $\mathbf{C}$.

A rigid body suspended by a compliant mechanism can achieve a general elastic behavior. A compliant mechanism consists of elastic components connected in different ways. Fig. 1 illustrates two types of simple compliant mechanisms. In a serial mechanism (Fig. 1a), each joint is loaded with a joint compliance; in a parallel mechanism (Fig. 1 b), each spring is connected independently to the suspended body. For both types of mechanisms, each joint compliance/stiffness can be obtained with a conventional torsional/translational spring or can be controlled in real-time using variable stiffness actuation (VSA) [3]. Thus, mechanisms considered are compliant mechanisms with lumped compliances. The elastic behavior of a compliant mechanism depends on its configuration and the value of compliance/stiffness of each joint/spring. As such, Cartesian compliance synthesis (or realization) requires identification of both the mechanism configuration and the compliance/stiffness of each joint/spring. In many robotic tasks, the configuration of the manipulator is an important concern due to physical constraints. Identification of the compliance realization conditions (both on the configuration and on the elastic component properties) is the primary motivation for this work. In addition, a better understanding and interpretation of these conditions for relatively simple compliant mechanisms yields a new means of compliant mechanism synthesis and provides insight into the design of hybrid serial/parallel compliant mechanisms.

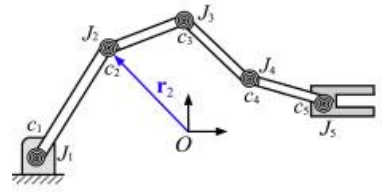

(a)

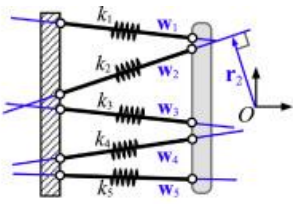

(b)

Fig. 1. Simple redundant compliant mechanisms with five components. (a) A 5-joint serial mechanism with joint compliances $c_{i} \geq 0$. The location of each joint $J_{i}$ is presented by a position vector $\boldsymbol{r}_{\mathrm{i}}$. (b) A 5 -spring parallel mechanism with spring rates $k_{i} \geq 0$. The axis of each spring is represented by wrench $\boldsymbol{w}_{\mathrm{i}}$ and $\boldsymbol{r}_{\mathrm{i}}$ is the perpendicular vector from the frame to $\boldsymbol{w}_{\text {i. }}$

In the design of a compliant mechanism, the space of realizable compliances with the mechanism is an important consideration. Since variable stiffness actuators enable joint stiffness to vary in real-time, the realizable space of compliances is significantly increased. However, as shown in [4], [5], [6], an arbitrary compliance cannot be achieved by varying the joint compliance/stiffness alone. The realizable space of compliant behaviors is highly restricted by the mobility of the mechanism. In order to further increase the mechanism mobility and enlarge the realizable space, mechanisms with kinematic redundancy can be used. Due to the increase in degrees of freedom, a redundant serial manipulator can reach an even larger space of compliances because the configuration of the mechanism can vary without affecting the pose of the endeffector. 


\subsection{Related work}

Many researchers have studied general spatial compliant behaviors. Screw theory [7], [8], [9], [10], [11] and Lie groups [12] have been widely used in spatial compliance analysis.

In previous work in spatial compliance realization, the conditions on stiffness/compliance that can be passively realized by simple parallel/serial compliant mechanisms have been identified [13], [14]. Synthesis procedures for any stiffness/compliance that is realizable with a simple compliant mechanism have been developed and refined [13], [14], [15], [16]. The realization of an arbitrary spatial compliance/stiffness using more complicated compliant components (helical joints in a serial mechanism or screw springs in a parallel mechanism) has also been addressed [17], [18], [19], [20]. In these approaches, the compliant behavior realization depends on a compliance/stiffness matrix decomposition into rank-1 components. In each process, mechanism geometry was not considered or constrained.

In more recent research, spatial compliance realization procedures that take into account some mechanism geometric properties have been developed [21], [22], [23], [24]. In our most recent work in spatial compliance realization [25], a geometry-based approach to realizing an arbitrary compliance was presented. In the synthesis procedure, each elastic component is selected from a restricted space based on its location, direction, and/or pitch.

In recent work on the realization of planar compliance, achieving a specified planar translational compliance (point compliance in Euclidean space $E(2)$ ) using a $3 R$ serial mechanism having given geometry has been addressed [26]. In the approach, an optimization was used. In [27], [28], methods to achieve an isotropic compliance in 2- and 3-dimensional Euclidean spaces with a simple serial mechanism were presented. In [4], conditions on mechanism geometry to attain every point compliance in $E(2)$ were identified, and synthesis procedures to achieve any given compliance in $E(2)$ using a 3 revolute-joint mechanism with given link lengths were developed. In [5], the results for $3 R$ mechanisms [4] were extended to general 3-joint mechanism (containing revolute and prismatic joints) having given (constrained) geometry.

In [29], a general $3 \times 3$ planar compliance (in SE(2)) synthesis procedure for the design of a 4-spring parallel mechanism having a specific symmetric structure was developed. In [6], a geometric synthesis procedure for a general planar compliance with a 3-component (non-redundant) compliant mechanism was presented. Most recently, compliance realization with a redundant mechanism consisting of 4 compliant components was addressed [30]. Realization conditions on the mechanism configuration to achieve a given compliance was identified. Synthesis procedures based on the mechanism geometry were developed.

\subsection{Contributions of the paper}

The limitations of prior work are best expressed in terms of serial mechanisms. The main limitation of previous work for $3 \mathrm{~J}$ and $4 \mathrm{~J}$ mechanisms is that the realizable space of compliant behaviors achieved in each mechanism is very limited. It is known that the space of all passive planar compliances is a 6 -dimensional cone (the $3 \times 3$ PSD cone). The space of compliances that can be achieved at a configuration of a $n$-joint mechanism is a $n$ dimensional polyhedral subcone. Although an arbitrary compliance can be realized with a $3 \mathrm{~J}$ or $4 \mathrm{~J}$ mechanism by properly choosing the joint locations, the set of acceptable joint locations is highly constrained.

When a $3 R$ mechanism is considered for the realization of a compliance, the realization conditions impose three equality constraints on the mechanism configuration [6], i.e., a wrench passing through any two joint locations must yield a twist centered at the location of the third joint [6]. Because a $3 R$ mechanism does not have redundancy, there is a unique mechanism configuration when the locations of the mechanism base and endeffector are specified. Since the realization conditions on $3 \mathrm{~J}$ mechanism are extremely restrictive [6], it is highly 
unlikely (if not impossible) that a desired compliance can be achieved at a specified endpoint pose, even with infinite variability in joint elastic behavior.

When a redundant mechanism consisting of 4 compliant components is considered (which increases the dimension of the realizable compliance space at a configuration by one), the realization conditions are still highly restrictive. Geometrically, the realization conditions require that a wrench passing through two joints yields a twist centered on a line segment connecting the other two joints. If the base location of the mechanism and pose of end-effector are specified, the remaining two joints must satisfy two equality conditions and four inequality conditions simultaneously to achieve the desired compliance. Since the space constrained by the realization conditions is so small, an arbitrarily specified compliance is still unlikely to be achieved by the mechanism.

It can been seen that the limitations of $3 \mathrm{~J}$ and $4 \mathrm{~J}$ mechanisms for compliance realization are related to their limited number of controllable compliant components. Thus, an investigation into compliance realization using mechanisms with additional compliant components is needed. Because the space of planar compliance is 6 dimensional, increasing the number of components to 5 will increase the dimension of the space of realizable compliance behaviors.

This paper addresses planar compliance synthesis with a redundant mechanism having five compliant components. The approaches developed for mechanisms of three and four compliant components [6], [30] cannot be used directly for this case for the following reasons: (1) the realizable compliance space of a 5-component mechanism is not simply the union of compliance subspaces realized with all combinations of three and four components in the mechanism (the contributions of all components must be considered simultaneously); (2) although the constraints on compliance are less restrictive due to the reduced number of equality conditions, the number of inequality conditions for passive realization is increased; and (3) the geometric significance of each realization condition is completely different due to the increase of compliant components.

The main contributions of the paper are:

- identification of necessary and sufficient conditions for 5-component mechanisms to realize an arbitrary compliance;

- identification of the geometric significance of each realization condition and interpretation of these conditions in terms of mechanism geometry;

- development of geometry-based synthesis procedures for both 5-joint serial mechanisms and 5-spring parallel mechanisms for the realization of an arbitrary compliance.

\subsection{Overview}

This paper presents a geometric approach to the design of a redundant compliant mechanism having five compliant components that passively realizes an arbitrary planar compliance. Realization conditions on mechanism configurations for an arbitrarily given compliance are identified. Geometry based synthesis procedures are developed for both 5-joint serial and 5-spring parallel mechanisms. With these procedures, the realization of any given compliant behavior can be accomplished by choosing each elastic component based on its geometry from an allowable space. Section 2 provides some background needed for the realization of a compliance by a simple serial/parallel mechanism. In Section 3, realization conditions on the mechanism configuration to achieve a given compliance are identified. In Section 4, the geometric significance of each realization condition is presented. In Section 5 , geometry based synthesis procedures are developed for both types of mechanisms. In Section 6 , numerical examples are presented to demonstrate the synthesis process. A brief conclusion and summary are provided in Section 7. 


\section{Technical background}

In this section, some background needed for the realization of a planar compliant behavior with a simple serial/parallel mechanism is presented. For each type of mechanism, only simple compliant components (no helical joints or screw springs) are needed.

\subsection{Screw representation of a mechanism configuration}

Consider a serial mechanism having $n$ joints $J_{i}(i=1,2, \ldots, n)$ as shown in Fig. 1a. Each joint can be described by a planar unit twist (the joint twist). In Plücker axis coordinates, the joint twists associated with a revolute joint $J_{\tau}$ and prismatic joint $J_{\rho}$ are:

(1) $\mathbf{t}_{\tau}=\left[\begin{array}{l}\mathbf{u} \\ 1\end{array}\right], \mathbf{t}_{\rho}=\left[\begin{array}{l}\widehat{\mathbf{n}} \\ 0\end{array}\right]$,

where $\mathbf{u}=\mathbf{r} \times \hat{\mathbf{k}}, \mathbf{r}$ is the position vector of $J_{\tau}, \hat{\mathbf{k}}$ is the unit vector orthogonal to the mechanism plane, and $\widehat{\mathbf{n}}$ is the (unit) vector along the axis of $J_{\rho}$.

If a twist $\mathbf{t}_{\tau}$ is specified, the center of the twist, $T_{\tau}$, can be calculated using

(2) $\mathbf{r}=\mathrm{Su}$

where $\mathbf{S} \in \mathbb{R}^{2 \times 2}$ is the skew-symmetric matrix:

(3) $\mathbf{S}=\left[\begin{array}{cc}0 & -1 \\ 1 & 0\end{array}\right]$.

Thus, once a joint twist $\mathbf{t}_{\mathbf{i}}$ for a revolute joint is identified, the location of the joint $J_{i}=T_{i}$ is uniquely determined as illustrated in Fig. 2a. Since a twist $\mathbf{t}_{\rho}$ associated with a prismatic joint has infinite pitch (free vector), it only determines the direction of the prismatic axis. The joint location is arbitrary in the plane.

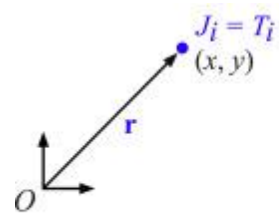

(a)

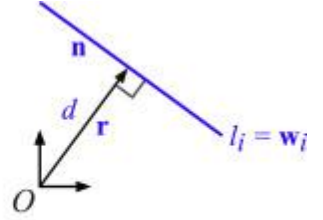

(b)

Fig. 2. Screw representation of the location of a mechanism component. (a) The location of joint $J_{i}$ in a serial mechanism can be represented by a unit twist $\mathbf{t}_{i}$. (b) The axis of a spring in a parallel mechanism is represented by a unit wrench $\mathbf{w}_{i}$.

For the parallel mechanism shown in Fig. 1b, each spring can be described by a planar unit wrench (the spring wrench). In Plücker ray coordinates, the spring wrenches corresponding to a line (translational) spring and a torsional spring are:

(4) $\mathbf{w}_{\rho}=\left[\begin{array}{l}\widehat{\mathbf{n}} \\ d\end{array}\right], \mathbf{w}_{\tau}=\left[\begin{array}{l}0 \\ 1\end{array}\right]$,

where $\widehat{\mathbf{n}}$ is a (unit) vector along the axis of spring, $d=(\mathbf{r} \times \widehat{\mathbf{n}}) \cdot \hat{\mathbf{k}}$,, and $\mathbf{r}$ is the orthogonal vector from frame $O$ to the spring axis as shown in Fig. 2 b.

If a wrench $\mathbf{w}_{\rho}$ is specified, the location of its axis (the line of action $l$ ) is determined by the perpendicular vector $\mathbf{r}$, which can be determined using

(5) $\mathbf{r}=-d \mathbf{S} \widehat{\mathbf{n}}$, 
where matrix $\mathbf{S}$ is defined in Eq. (3).

Thus, once a spring wrench $\mathbf{w}_{\rho}$ associated with a line spring is identified, the axis of the spring $l$ is uniquely determined. Since the spring wrench $\mathbf{w}_{\tau}$ in Eq. (4) has infinite pitch, a torsional spring can be located anywhere in the plane.

\subsection{Screw product of a twist and wrench}

Since the center of a twist $\mathbf{t}, T$, is independent of its density (magnitude), a scalar multiplication of a twist does not change its location. Similarly, a scalar multiplication of a wrench does not change its line of action $l$. For planar cases, a point $T$ is uniquely identified by a unit twist $\hat{\mathbf{t}}$. A line $l$ is uniquely identified by a unit wrench $\widehat{\mathbf{w}}$.

The screw product (reciprocal product) of a twist $\mathbf{t}$ and a wrench $\mathbf{w}$, if expressed in Plücker axis and ray coordinates respectively, is defined as

$\mathbf{w}^{T} \mathbf{t}=\mathbf{t}^{T} \mathbf{w}$

which indicates the work done by the wrench along the twist.

If $\mathbf{t}$ is a finite pitch unit twist expressed in Eq. (1) and $\mathbf{w}$ is a finite pitch unit wrench expressed in Eq. (4), then the reciprocal product of $\mathbf{t}$ and $\mathbf{w}$ indicates the distance from the twist center of $\mathbf{t}$ to the axis of $\mathbf{w}$, i.e., if a point $T$ is represented by a unit twist $\mathbf{t}$ and a line $l$ is represented by a unit wrench $\mathbf{w}$, then the distance from $T$ to $l$ is calculated by

(6) $d=\mathbf{w}^{T} \mathbf{t}=\mathbf{t}^{T} \mathbf{w}$.

A twist $\mathbf{t}$ and a wrench $\mathbf{w}$ are called reciprocal [7] if the screw product of the two is zero:

(7) $\mathbf{w}^{T} \mathbf{t}=\mathbf{t}^{T} \mathbf{w}=0$,

which indicates that the wrench $\mathbf{w}$ does no work along twist $\mathbf{t}$.

For planar cases, the reciprocal relationship of a twist $\mathbf{t}$ and wrench $\mathbf{w}$ can be represented geometrically if both have finite pitches. If $T$ is the center of $\mathbf{t}$ and $l$ is the axis of $\mathbf{w}$, then $\mathbf{t}$ and $\mathbf{w}$ are reciprocal if and only if $T$ is on $l$. Note that the reciprocal product of two screws is coordinate frame independent.

\subsection{Compliance achieved by a passive elastic mechanism}

Consider a $n$-joint serial mechanism. Each joint has joint twists $\mathbf{t}_{i}$ and joint compliance $c_{i} \geq 0(i=1, \ldots, n)$. The Cartesian compliance $\mathbf{C}$ at the configuration is [18]:

(8) $\mathbf{C}=c_{1} \mathbf{t}_{1} \mathbf{t}_{1}^{T}+c_{2} \mathbf{t}_{2} \mathbf{t}_{2}^{T}+\cdots+c_{n} \mathbf{t}_{n} \mathbf{t}_{n}^{T}$.

This equation can be express in the form:

(9) $\mathbf{C}=\mathbf{T C}_{J} \mathbf{T}^{T}$,

where $\mathbf{T}=\left[\mathbf{t}_{1}, \mathbf{t}_{2}, \ldots, \mathbf{t}_{n}\right]$ and $\mathbf{C}_{J}$ is the (diagonal) joint compliance matrix $\mathbf{C}_{J}=\operatorname{diag}\left[c_{1}, c_{2}, \ldots, c_{n}\right]$. Since each column of $\mathbf{T}$ presents a unit twist contributed by a joint, $\mathrm{T}$ is the Jacobian of the serial mechanism. Thus, the realization of Cartesian compliance matrix $\mathbf{C}$ requires that an appropriate set of joint twists $\mathbf{t}_{i}$ (or the mechanism Jacobian) and corresponding joint compliances $c_{i}$ are identified. In general, due to the non-uniqueness of the decomposition in Eq. (8), there is an infinite number of mechanism configurations that can realize a given $\mathbf{C}$.

Dual to serial mechanism realization, for a parallel mechanism having spring wrenches $\mathbf{w}_{i}$ and spring stiffnesses $k_{i} \geq 0(i=1, \ldots, n)$,the Cartesian stiffness $\mathbf{K}[17]$ is: 


$$
(10) \mathbf{K}=k_{1} \mathbf{w}_{1} \mathbf{w}_{1}^{T}+\cdots+k_{n} \mathbf{w}_{n} \mathbf{w}_{n}^{T} .
$$

Similar to the serial case, Eq. (10) can be expressed as

$$
(11) \mathbf{K}=\mathbf{W} \mathbf{K}_{J} \mathbf{W}^{T} \text {, }
$$

where $\mathbf{W}=\left[\mathbf{w}_{1}, \mathbf{w}_{2}, \ldots, \mathbf{w}_{n}\right]$ and $\mathbf{K}_{J}$ is the diagonal joint stiffness matrix $\mathbf{K}_{J}=\operatorname{diag}\left[k_{1}, k_{2}, \ldots, k_{n}\right]$. Since each column of $\mathbf{W}$ presents a unit wrench imposed by each spring, $\mathbf{W}^{T}$ is the Jacobian of the parallel mechanism. Thus, the realization of Cartesian stiffness $\mathbf{K}$ requires that an appropriate set of spring wrenches $\mathbf{w}_{i}$ (or the mechanism Jacobian) and the corresponding spring stiffnesses $k_{i} \geq 0$ are identified.

Note that Eq. (8) for the Cartesian compliance of a serial mechanism and Eq. (10) for the Cartesian stiffness of a parallel mechanism each applies for a general $n$-component mechanism (for both redundant and non-redundant mechanisms). It can be seen that if used for the realization of a full-rank $3 \times 3$ compliance or stiffness matrix, a mechanism must have at least 3 components $(n \geq 3)$. Due to the rank deficiency of a prismatic joint twist and a torsional spring wrench, a serial mechanism containing prismatic joints alone can only attain a rank-2 matrix, and a parallel mechanism containing only torsional springs can only attain a rank-1 matrix. Hence, if a serial mechanism is used to realize a full-rank compliance matrix, it must have at least one revolute joint; and if a parallel mechanism is used to realize an arbitrary stiffness, at least two line springs must be used. Realization of a given compliance requires conditions on the locations of revolute joints (represented by joint twists) of a serial mechanism, and conditions on the spring axes (represented by spring wrenches) of a parallel mechanism. Since each joint twist $\mathbf{t}_{i}$ and each spring wrench $\mathbf{w}_{i}$ has geometric meaning, using screw representation, mathematical conditions for compliance realization can be interpreted in terms of mechanism geometry and expressed graphically in the plane.

\section{Realization conditions}

In this section, realization conditions for a 5-component compliant mechanism to achieve a given compliance are identified. Serial mechanisms are considered first. Then, by duality, the results are modified and extended to parallel mechanisms.

\subsection{Realization conditions for 5 -joint serial mechanisms}

Consider a 5 -joint serial mechanism. Each joint $J_{i}$ is represented by joint twist $\mathbf{t}_{i}(i=1,2, \ldots, 5)$. A line that passes through two joints $J_{i}$ and $J_{i}$ (represented by a wrench $\mathbf{w}_{\mathrm{ij}}$ ) satisfies the reciprocal conditions for both $\mathbf{t}_{i}$ and $\mathbf{t}_{j}$ :

$$
(12) \mathbf{w}_{i j}^{T} \mathbf{t}_{i}=0 \text { and } \mathbf{w}_{i j}^{T} \mathbf{t}_{j}=0 \text {. }
$$

Since a scalar multiplication of a wrench does not change its axis, $\mathbf{w}_{\mathrm{ij}}$ can be calculated by

$$
\text { (13) } \mathbf{w}_{i j}=\alpha\left(\mathbf{t}_{i} \times \mathbf{t}_{j}\right), \forall i \neq j,
$$

where $\alpha$ is an arbitrary scalar.

Suppose that a symmetric matrix $\mathbf{C}$ is expressed as:

$$
(14) \mathbf{C}=c_{1} \mathbf{t}_{1} \mathbf{t}_{1}^{T}+c_{2} \mathbf{t}_{2} \mathbf{t}_{2}^{T}+c_{3} \mathbf{t}_{3} \mathbf{t}_{3}^{T}+c_{4} \mathbf{t}_{4} \mathbf{t}_{4}^{T}+c_{5} \mathbf{t}_{5} \mathbf{t}_{5}^{T},
$$

and suppose that wrench $\mathbf{w}_{12}$ passes through joints $J_{1}$ and $J_{2}$; and wrench $\mathbf{w}_{34}$ passes through joints $J_{3}$ and $J_{4}$. Then, using Eq. (12), 


$$
\begin{aligned}
\mathbf{C} \mathbf{w}_{12} & =\left(c_{1} \mathbf{t}_{1} \mathbf{t}_{1}^{T}+c_{2} \mathbf{t}_{2} \mathbf{t}_{2}^{T}+c_{3} \mathbf{t}_{3} \mathbf{t}_{3}^{T}+c_{4} \mathbf{t}_{4} \mathbf{t}_{4}^{T}+c_{5} \mathbf{t}_{5} \mathbf{t}_{5}^{T}\right) \mathbf{w}_{12} . \\
& =c_{3}\left(\mathbf{t}_{3}^{T} \mathbf{w}_{12}\right) \mathbf{t}_{3}+c_{4}\left(\mathbf{t}_{4}^{T} \mathbf{w}_{12}\right) \mathbf{t}_{4}+c_{5}\left(\mathbf{t}_{5}^{T} \mathbf{w}_{12}\right) \mathbf{t}_{5} .
\end{aligned}
$$

Applying Eq. (12) again to the above equation yields:

$$
\text { (15) } \mathbf{w}_{34}^{T} \mathbf{C} \mathbf{w}_{12}=c_{5}\left(\mathbf{t}_{5}^{T} \mathbf{w}_{12}\right)\left(\mathbf{t}_{5}^{T} \mathbf{w}_{34}\right) \text {. }
$$

Applying the same process with $\mathbf{w}_{13}$ and $\mathbf{w}_{24}$ yields:

$$
\text { (16) } \mathbf{w}_{24}^{T} \mathbf{C} \mathbf{w}_{13}=c_{5}\left(\mathbf{t}_{5}^{T} \mathbf{w}_{24}\right)\left(\mathbf{t}_{5}^{T} \mathbf{w}_{13}\right) \text {. }
$$

Solving both Eqs. (15) and $\underline{\underline{16})}$ for $c_{5}$ yields:

$$
\text { (17) } \frac{\mathbf{w}_{24}^{T} C \mathbf{w}_{13}}{\left(\mathbf{t}_{5}^{T} \mathbf{w}_{24}\right)\left(\mathbf{t}_{5}^{T} \mathbf{w}_{13}\right)}=\frac{\mathbf{w}_{34}^{T} C \mathbf{w}_{12}}{\left(\mathbf{t}_{5}^{T} \mathbf{w}_{12}\right)\left(\mathbf{t}_{5}^{T} \mathbf{w}_{34}\right)}
$$

In general, if the decomposition (14) applies to $\mathbf{C}$, then, for any permutation $(i, j, p, q, r)$ from $\{1,2,3,4,5\}$ :

$$
\text { (18) } \frac{\mathbf{w}_{i j}^{T} \mathbf{C} \mathbf{w}_{p q}}{\left(\mathbf{t}_{r}^{T} \mathbf{w}_{i j}\right)\left(\mathbf{t}_{r}^{T} \mathbf{w}_{p q}\right)}=\frac{\mathbf{w}_{i p}^{T} \mathbf{C} \mathbf{w}_{j q}}{\left(\mathbf{t}_{r}^{T} \mathbf{w}_{i p}\right)\left(\mathbf{t}_{r}^{T} \mathbf{w}_{j q}\right)}
$$

where $\mathbf{w}_{i j}$ is a wrench passing through the centers of $\mathbf{t}_{i}$ and $\mathbf{t}_{j}\left(U_{i}\right.$ and $\left.J_{j}\right)$ defined in Eqs. (12) or (13).

Thus, Eq. (18) is a necessary condition for $\mathbf{C}$ to be achieved at a configuration of a 5 -joint serial mechanism. Using a process similar to that presented in [30] for 4-component mechanisms, it can be shown that Eq. (18) is also a sufficient condition. Thus, if Eq. (18) is satisfied for one permutation, then it must be satisfied for all permutations; and if matrix $\mathrm{C}$ can be decomposed into the form of $\underline{(14)}$, then for every permutation $(i, j, p, q, r)$ from $\{1,2,3,4,5\}$,

$$
\text { (19) } c_{r}=\frac{\mathbf{w}_{i j}^{T} \mathbf{C w}_{p q}}{\left(\mathbf{w}_{i j}^{T} \mathbf{t}_{r}\right)\left(\mathbf{w}_{p q}^{T} \mathbf{t}_{r}\right)}=\frac{\mathbf{w}_{i p}^{T} \mathbf{C w}_{j q}}{\left(\mathbf{w}_{i p}^{T} \mathbf{t}_{r}\right)\left(\mathbf{w}_{j q}^{T} \mathbf{t}_{r}\right)} .
$$

Therefore, satisfaction of condition (18) for any one permutation $(i, j, p, q, r)$ of $\{1,2,3,4,5\}$ is a necessary and sufficient condition for $\mathrm{C}$ to be decomposed into the form of $(14)$. Note that in the decomposition of Eq. (14), no restriction on each coefficient $c_{i}$ is yet imposed. This means that if condition (18) is violated for one permutation, the mechanism cannot achieve the given $\mathbf{C}$ at the configuration even if each joint compliance can vary indefinitely in $(-\infty,+\infty)$.

For a PSD compliance matrix $\mathbf{C}$ to be realized passively (using conventional springs or variable stiffness actuation), each joint compliance must be nonnegative, which requires $c_{r} \geq 0$ in Eq. (19). It is readily shown that, if for any five permutations with $r$ being each of the joints $(r=1,2,3,4,5)$,

$\frac{\mathbf{w}_{i j}^{T} \mathbf{C} \mathbf{w}_{p q}}{\left(\mathbf{w}_{i j}^{T} \mathbf{t}_{r}\right)\left(\mathbf{w}_{p q}^{T} \mathbf{t}_{r}\right)} \geq 0$,

then, the inequality conditions must be satisfied for all permutations of $\{1,2,3,4,5\}$. Thus, the realization conditions can be summarized as:

\section{Proposition 1}

Suppose $\boldsymbol{t}_{\mathrm{i}}(\mathrm{i}=1,2, \ldots, 5)$ are the joint twists of a 5 -joint serial mechanism. A given compliance $\mathbf{C}$ can be achieved passively at the mechanism configuration if and only if the following conditions hold:

(i) For any one permutation $(i, j, p, q, r)$ of $\{1,2,3,4,5\}$, 


$$
\text { (20) } \frac{\mathbf{w}_{i j}^{T} \mathbf{C} \mathbf{w}_{p q}}{\left(\mathbf{t}_{r}^{T} \mathbf{w}_{i j}\right)\left(\mathbf{t}_{r}^{T} \mathbf{w}_{p q}\right)}=\frac{\mathbf{w}_{i p}^{T} \mathbf{C} \mathbf{w}_{j q}}{\left(\mathbf{t}_{r}^{T} \mathbf{w}_{i p}\right)\left(\mathbf{t}_{r}^{T} \mathbf{w}_{j q}\right)}
$$

(ii) For any five permutations with $r=1,2,3,4,5$,

$$
\text { (21) } \frac{\mathbf{w}_{i j}^{T} \mathbf{C} \mathbf{w}_{p q}}{\left(\mathbf{w}_{i j}^{T} \mathbf{t}_{r}\right)\left(\mathbf{w}_{p q}^{T} \mathbf{t}_{r}\right)} \geq 0
$$

Therefore, the realization of a compliance at a given configuration requires the satisfaction of one equality condition in the form of $\underline{(20)}$ and five inequality conditions in the form of $\underline{21)}$.

\subsection{Realization conditions for 5-spring parallel mechanisms}

By duality, a set of necessary and sufficient conditions on a 5-spring parallel mechanism to realize a given stiffness can be obtained.

Suppose that $\mathbf{w}_{i}((i=1,2, \ldots, 5))$ are the spring wrenches of a parallel mechanism. If two wrenches $\mathbf{w}_{i}$ and $\mathbf{w}_{j}$ intersect at $T_{i j}$, then the two wrenches must be reciprocal to a twist $\boldsymbol{t}_{\mathrm{ij}}$ centered at $T_{i j}$. Using the reciprocal condition, $\boldsymbol{t}_{\mathrm{ij}}$ can be calculated using

$$
(22) \mathbf{t}_{i j}=\beta\left(\mathbf{w}_{i} \times \mathbf{w}_{j}\right),
$$

where $\beta$ is an arbitrary scalar. With appropriate modification, the results of Proposition 1 obtained for a 5-joint serial mechanism apply to a 5-spring parallel mechanism for the realization of an arbitrary stiffness.

\section{Proposition 2}

Suppose $\left.\mathbf{w}_{i}(i=1, \ldots, 5)\right)$ are spring wrenches of a 5-spring parallel mechanism. A given stiffness $\boldsymbol{K}$ can be achieved passively by the mechanism if and only if the following conditions hold:

(i) For an arbitrary permutation $(i, j, p, q, r)$ of $\{1,2,3,4,5\}$,

$$
\text { (23) } \frac{\mathbf{t}_{i j}^{T} \mathbf{K t}_{p q}}{\left(\mathbf{t}_{i j}^{T} \mathbf{w}_{r}\right)\left(\mathbf{t}_{p q}^{T} \mathbf{w}_{r}\right)}=\frac{\mathbf{t}_{i p}^{T} \mathbf{K t}_{j q}}{\left(\mathbf{t}_{i p}^{T} \mathbf{w}_{r}\right)\left(\mathbf{t}_{j q}^{T} \mathbf{w}_{r}\right)} .
$$

(ii) For any five permutations of $(i, j, p, q, r)$ of $\{1,2,3,4,5\}$ with $r=1,2,3,4,5$,

$$
\text { (24) } \frac{\mathbf{t}_{i j}^{T} \mathbf{K t}_{p q}}{\left(\mathbf{t}_{i j}^{T} \mathbf{w}_{r}\right)\left(\mathbf{t}_{p q}^{T} \mathbf{w}_{r}\right)} \geq 0
$$

Dual to the serial case, if Eq. (23) holds for any one permutation, then it must hold for all permutations. If inequality $\underline{(24)}$ holds for any five permutations (with $s=1,2,3,4,5)$, then the inequality must hold for all permutations. Thus, to realize a given stiffness using a 5-spring parallel mechanism, one equality condition in the form of $\underline{(23)}$ and five inequality conditions in the form of $\underline{(24)}$ must be satisfied.

\subsection{The uniqueness of the realization}

If at a given configuration, a $5 \mathrm{~J}$ serial mechanism realizes a compliance, the realization is typically unique. This is due to the fact that, if among the five joint twists no three are linearly dependent (the generic case), then each joint compliance $c_{r}$ calculated by Eq. (19) is unique regardless of the permutation used in the calculation. Geometrically, the independence of three joint twists indicates that the locations of the three joints corresponding to these twists are not located on a single line. Since the mechanism Jacobian is full-rank for this generic case, the realization is unique for any non-singular configuration. It is easy to see that, at a singular configuration, the denominators of some $c_{i}$ in Eq. (24)are zero. The joint compliance of joint $J_{r}$ can be uniquely determined with $(i, j, p, q)$ being any permutation of $\{1,2,3,4,5\}$ excluding $r$, i.e., $(i, j, p, q)=\{1,2,3,4,5\} \backslash r$ : 


$$
\text { (25) } c_{r}=\frac{\mathbf{w}_{i j}^{T} \mathbf{C} \mathbf{w}_{p q}}{\left(\mathbf{w}_{i j}^{T} \mathbf{t}_{r}\right)\left(\mathbf{w}_{p q}^{T} \mathbf{t}_{r}\right)} \text {. }
$$

Although different permutations can be used in calculating $c_{r}$ using Eq. (25), condition (ii) of Proposition 1 ensures that the joint compliance $c_{r}$ obtained is the same for the given set of joint twists at the configuration.

Similarly, if a 5-spring parallel mechanism realizes a stiffness, and if among the five spring wrenches, no three are linearly dependent, then each spring stiffness $k_{r}$ in the realization must be unique. Geometrically, the independence of three spring wrenches indicates that the three wrenches do not intersect at a single point or are not parallel to each other. For this case, the mechanism Jacobian $\mathbf{W}^{T}$ in Eq. (11) is full-rank (has rank 3). Thus, for a non-singular configuration, each spring stiffness can be uniquely obtained with an arbitrary permutation $(i, j, p, q)=\{1,2,3,4,5\} \backslash r$ :

(26) $k_{r}=\frac{\mathbf{t}_{i j}^{T} \mathbf{K t}_{p q}}{\left(\mathbf{t}_{i j}^{T} \mathbf{w}_{r}\right)\left(\mathbf{t}_{p q}^{T} \mathbf{w}_{r}\right)}$

\section{Geometric significance of the realization conditions}

The set of realization conditions developed in Section 3 can be viewed in terms of the mechanism geometry. Since the realization conditions involve the product of two wrenches/twists about a given compliance/stiffness in Eq. (20) or (23), the physical significance of just the two products is identified first. Then, the geometric interpretations of all of the realization conditions are provided for the two types of simple 5-component mechanisms.

\subsection{Screw product about compliance}

As stated in Section 2.2, if a twist $\mathbf{t}$ and wrench $\mathbf{w}$ are both unit screws, then the screw product of $\mathbf{t}$ and $\mathbf{w}$ indicates the distance from the twist instantaneous center $T$ to the wrench axis $l$. Below, we identify the physical significance of the product of two unit wrenches about a given compliance $\mathbf{C}$.

Consider two unit wrenches $\mathbf{w}_{1}$ and $\mathbf{w}_{2}$. We show that, for a given compliance matrix $\mathbf{C}$, the quantity

$$
\text { (26) } h=\mathbf{w}_{2}^{T} \mathbf{C} \mathbf{w}_{1}
$$

is related to the location of the compliance center.

Note that $h$ defined in Eq. (27) is invariant under coordinate transformation for $\mathbf{w}_{1}, \mathbf{w}_{2}$ and $\mathbf{C}$. To clearly show the physical significance of $h$, a coordinate frame located at the compliance center $C_{c}$ is used initially. In this coordinate frame, the compliance matrix $\mathrm{C}$ has the block diagonal form:

$$
(27) \mathbf{C}_{n}=\left[\begin{array}{cc}
\mathbf{D} & \mathbf{0} \\
\mathbf{0}^{T} & c_{\phi}
\end{array}\right]
$$

where $\mathbf{D}$ is a $2 \times 2$ symmetric PSD matrix, $\mathbf{0}$ is the zero 2 -vector, and $c_{\phi}>0$ is the principal rotational compliance of $\mathbf{C}$. Note that with the compliance matrix expressed in this form, the value of $c_{\phi}$ is unique.

In the compliance center based frame, an arbitrary unit wrench $\mathbf{w}_{1}$ has the form:

$$
\text { (28) } \mathbf{w}_{1}=\left[\begin{array}{l}
\mathbf{n}_{1} \\
d_{1}^{c}
\end{array}\right],
$$

where $d_{1}^{c}$ is the distance from the axis of $\mathbf{w}_{1}$ to the frame origin, i.e., to the center of compliance $C_{c}$. The twist corresponding to $w_{1}$ is: 


$$
(29) \mathbf{t}_{1}=\mathbf{C}_{n} \mathbf{w}_{1}=\left[\begin{array}{cc}
\mathbf{D} & \mathbf{0} \\
\mathbf{0}^{T} & c_{\phi}
\end{array}\right]\left[\begin{array}{l}
\mathbf{n}_{1} \\
d_{1}^{c}
\end{array}\right]=\left[\begin{array}{c}
\mathbf{D} \mathbf{n}_{1} \\
c_{\phi} d_{1}^{c}
\end{array}\right]
$$

The unit twist associated with $\mathbf{t}_{1}$ is:

$$
\text { (30) } \hat{\mathbf{t}}_{1}=\frac{1}{c_{\phi} d_{1}^{c}} \mathbf{t}_{1}
$$

which has the same center as $t_{1}$. Premultiplying $t_{1}$ by unit wrench $\mathbf{w}_{2}$ yields:

$$
\text { (31) } \mathbf{w}_{2}^{T} \mathbf{t}_{1}=\mathbf{w}_{2}^{T} \mathbf{C}_{n} \mathbf{w}_{1}=\left(c_{\phi} d_{1}^{C}\right)\left(\mathbf{w}_{2}^{T} \hat{\mathbf{t}}_{1}\right) \text {. }
$$

Since $\hat{\mathbf{t}}_{1}$ is a unit twist and $\mathbf{w}_{2}$ is a unit wrench, $\left(\mathbf{w}_{2}^{T} \hat{\mathbf{t}}_{1}\right)$ indicates the distance from the center of $\mathbf{t}_{1}, T_{1}$, to the wrench axis of $\mathbf{w}_{2}$. Thus,

$$
(32) \mathbf{w}_{2}^{T} \mathbf{C}_{n} \mathbf{w}_{1}=c_{\phi}\left(\mathbf{w}_{1}^{T} \mathbf{t}_{c}\right)\left(\mathbf{w}_{2}^{T} \hat{\mathbf{t}}_{1}\right)=c_{\phi} d_{1}^{c} d_{2}^{1},
$$

where $\mathbf{t}_{c}$ is the unit twist located at the compliance center $C_{c}, d_{1}^{c}$ indicates the distance from $C_{c}$ to the wrench

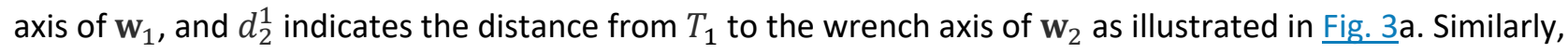

$$
\text { (33) } \mathbf{w}_{1}^{T} \mathbf{C}_{n} \mathbf{w}_{2}=c_{\phi} d_{2}^{c} d_{1}^{2},
$$

where $d_{2}^{c}$ indicates the distance from $C_{c}$ to the wrench axis of $\mathbf{w}_{2}, d_{2}^{1}$ indicates the distance from $T_{2}$ to the wrench axis of $\mathbf{w}_{1}$ (illustrated in Fig. 3 b). Since the products in Eqs. (33) and (34) are frame independent, and since $\mathbf{C}$ is symmetric, in an arbitrary frame,

$$
\text { (34) } \mathbf{w}_{1}^{T} \mathbf{C w}_{2}=\mathbf{w}_{2}^{T} \mathbf{C w}_{1}=c_{\phi} d_{1}^{c} d_{2}^{1}=c_{\phi} d_{2}^{c} d_{1}^{2} .
$$

Thus, the product $h$ defined in Eq. (27) is related to the rotational principal compliance of $\mathbf{C}$ and the positions of $\mathbf{w}_{i}$ relative to the compliance center.

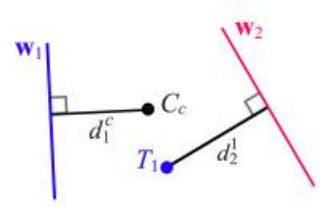

(a)

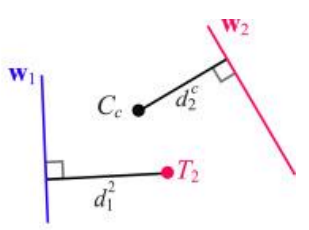

(b)

Fig. 3. Geometric significance of the product of two wrenches about $\mathbf{C}$.

In a dual development, the product $\mathbf{t}_{2} \mathbf{K t}_{1}$ is invariant under coordinate transformation for $\mathbf{t}_{1}, \mathbf{t}_{2}$ and $\mathbf{K}$. When expressed in a frame located at its stiffness center $C_{k}$, a stiffness $\mathbf{K}$ has the following block diagonal form:

$$
(35) \mathbf{K}_{n}=\left[\begin{array}{cc}
\mathbf{A} & \mathbf{0} \\
\mathbf{0}^{T} & k_{\phi}
\end{array}\right],
$$

where $k_{\phi}>0$ is the principal rotational stiffness of $\mathbf{K}$ and is uniquely determined by the stiffness behavior.

Suppose that $\mathbf{t}_{1}$ and $\mathbf{t}_{2}$ are two unit twists, and that, in the coordinate frame located at $C_{k}$, the two twists have the form:

$$
(36) \mathbf{t}_{i}=\left[\begin{array}{c}
\mathbf{u}_{i} \\
1
\end{array}\right], i=1,2
$$

Then, the wrench associated with $\mathbf{t}_{1}$ acting on $\mathbf{K}_{\mathrm{n}}$ is:

$$
\text { (37) } \mathbf{w}_{1}=\mathbf{K}_{n} \mathbf{t}_{1}=\left[\begin{array}{cc}
\mathbf{A} & \mathbf{0} \\
\mathbf{0}^{T} & k_{\phi}
\end{array}\right]\left[\begin{array}{c}
\mathbf{u}_{1} \\
1
\end{array}\right]=\left[\begin{array}{c}
\mathbf{A} \mathbf{u}_{1} \\
k_{\phi}
\end{array}\right]
$$


The unit wrench associated with $\mathbf{w}_{1}$ is:

$$
\text { (38) } \widehat{\mathbf{w}}_{1}=\frac{1}{\left\|\mathrm{Au}_{1}\right\|} \mathbf{w}_{1},
$$

The third component of $\widehat{w}_{1}$ is the distance from the wrench axis to the frame origin, which is at the stiffness center $C_{k}$, i.e.,

$$
\text { (39) } d_{1}^{c}=\frac{k_{\phi}}{\left\|\mathbf{A u}_{1}\right\|} \text {. }
$$

Thus,

$$
(40) \mathbf{t}_{2}^{T} \mathbf{K}_{n} \mathbf{t}_{1}=\mathbf{t}_{2}^{T} \mathbf{w}_{1}=\left\|A \mathbf{u}_{1}\right\|\left(\mathbf{t}_{2}^{T} \widehat{\mathbf{w}}_{1}\right) .
$$

Using Eq. (40),

$\mathbf{t}_{2}^{T} \mathbf{K}_{n} \mathbf{t}_{1}=k_{\phi} \frac{\left(\mathbf{t}_{2}^{T} \widehat{\mathbf{w}}_{1}\right)}{d_{1}^{c}}$

Since $\mathbf{t}_{2}$ and $\widehat{\mathbf{w}}_{1}$ are both unit screws, $\mathbf{t}_{2}^{T} \widehat{\mathbf{w}}_{1}$ indicates the distance from $T_{2}$ (the twist center of $\mathbf{t}_{2}$ ) to the wrench axis of $\mathbf{w}_{1}$. Since the product in Eq. (41) is frame independent, in an arbitrary coordinate frame,

$$
\text { (41) } \mathbf{t}_{2}^{T} \mathbf{K} \mathbf{t}_{1}=k_{\phi} \frac{\mathbf{t}_{2}^{T} \mathbf{w}_{1}}{\mathbf{w}_{1}^{T} \mathbf{t}_{c}}
$$

where $\mathbf{t}_{c}$ is the unit twist located at the center of stiffness. Reversing the order of multiplication yields:

$$
(42) \mathbf{t}_{1}^{T} \mathbf{K t}_{2}=k_{\phi} \frac{\mathbf{t}_{1}^{T} \mathbf{w}_{2}}{\mathbf{w}_{2}^{T} \mathbf{t}_{c}} .
$$

Since $\mathrm{K}$ is symmetric, Eqs. (42) and (43) are equal and can be expressed in terms of geometric parameters:

$$
\text { (43) } \mathbf{t}_{1}^{T} \mathbf{K} \mathbf{t}_{2}=\mathbf{t}_{2}^{T} \mathbf{K} \mathbf{t}_{1}=k_{\phi} \frac{d_{1}^{2}}{d_{1}^{c}}=k_{\phi} \frac{d_{2}^{1}}{d_{2}^{c}}
$$

where $d_{1}^{2}$ indicates the distance from $T_{2}$ to the wrench axis of $\mathbf{w}_{1}, d_{1}^{c}$ indicates the distance from $C_{k}$ to the wrench axis of $\mathbf{w}_{1}$ as illustrated in Fig. $4 \mathrm{a}$; and where $d_{2}^{1}$ is the distance from $T_{1}$ to the wrench axis of $\mathbf{w}_{2}, d_{2}^{c}$ indicates the distance from $C_{k}$ to the wrench axis of $\mathbf{w}_{2}$ as illustrated in Fig. $4 \mathrm{~b}$.

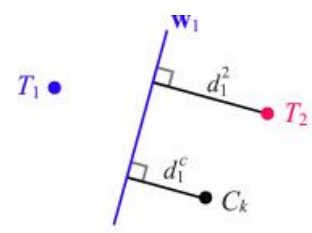

(a)

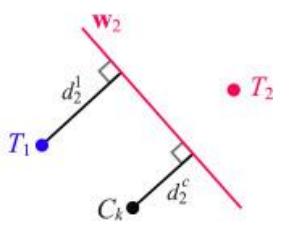

(b)

Fig. 4. Geometric significance of the product of two twists about K.

\subsection{Geometric significance of the realization conditions for serial mechanisms}

For a 5 -joint mechanism with joint twists $\mathbf{t}_{i}(i=1,2, \ldots, 5)$,, let $\mathbf{w}_{i j}$ be the unit wrench reciprocal to twists $\mathbf{t}_{i}$ and $\mathbf{t}_{j}$, then the axis of $\mathbf{w}_{i j}$ passes through the two joints, $J_{i}$ and $J_{j}$. Denote:

$\mathbf{t}_{i j}=\mathbf{C w}_{i j}$.

Then, $\mathbf{w}_{i j}$ and $\boldsymbol{t}_{\mathrm{ij}}$ are solely determined by the mechanism geometry and the desired compliance $\mathbf{C}$. The realization conditions require that one equality condition in the form of $\underline{(20)}$ is satisfied and five inequality 
conditions in the form of $\underline{21)}$ are satisfied. Below, the geometric significance of each of these conditions is identified.

\subsubsection{Equality condition for serial mechanisms}

Using the results presented in Section 4.1, the geometric significance of equality condition (20) can be obtained. Here, we consider the case $[i, j, p, q, r]=[1,2,3,4,5]$. The equality realization condition $(20)$ for this case requires that

$$
\text { (44) } \frac{\mathbf{w}_{12}^{T} \mathbf{C w}_{34}}{\left(\mathbf{w}_{12}^{T} \mathbf{t}_{5}\right)\left(\mathbf{w}_{34}^{T} \mathbf{t}_{5}\right)}=\frac{\mathbf{w}_{23}^{T} \mathbf{C w}_{14}}{\left(\mathbf{w}_{23}^{T} \mathbf{t}_{5}\right)\left(\mathbf{w}_{14}^{T} \mathbf{t}_{5}\right)} .
$$

Using Eq. (35), this can be expressed in geometric terms as:

$c_{\phi} \frac{d_{12}^{c} d_{34}^{12}}{d_{12}^{5} d_{34}^{5}}=c_{\phi} \frac{d_{23}^{c} d_{14}^{23}}{d_{23}^{5} d_{14}^{5}}$.

Since $c_{\phi}$ is constant for a given compliance, the condition is reduced to only geometric terms:

$$
\text { (45) } \frac{d_{12}^{c} d_{34}^{12}}{d_{12}^{5} d_{34}^{5}}=\frac{d_{23}^{c} d_{14}^{23}}{d_{23}^{5} d_{14}^{5}}
$$

where $d_{12}^{c}$ is the distance from the compliance center $C_{c}$ to line $J_{1} J_{2}, d_{34}^{12}$ is the distance from the center of $\mathbf{t}_{12}$ to line $J_{3} J_{4}, d_{12}^{5}$ is the distance from $J_{5}$ to line $J_{1} J_{2}$, and $d_{34}^{5}$ is the distance from $J_{5}$ to line $J_{3} J_{4}$ (each illustrated in Fig. 5a); and where $d_{23}^{c}$ is the distance from the compliance center $C_{c}$ to line $J_{2} J_{3}, d_{14}^{23}$ is the distance from the center of $\mathbf{t}_{23}$ to line $J_{1} J_{4}, d_{23}^{5}$ is the distance from $J_{5}$ to line $J_{2} J_{3}$, and $d_{14}^{5}$ is the distance from $J_{5}$ to line $J_{1} J_{4}$ (each illustrated in Fig. $5 \mathrm{~b}$ ). The equality condition $(46)$ requires that the ratios between the distances in Fig. $5 \mathrm{a}$ and $\underline{5} \mathrm{~b}$ must be the same. Thus, the equality realization condition is expressed in terms of the mechanism geometry and compliance properties.

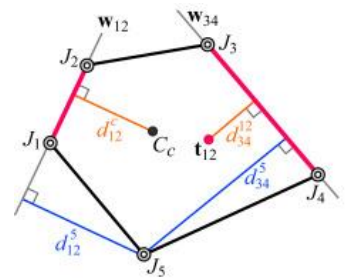

(a)

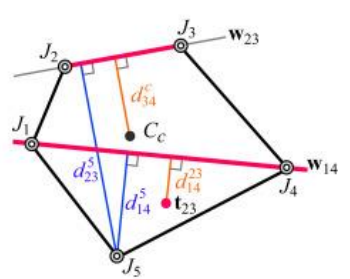

(b)

Fig. 5. Geometric interpretation on the equality condition for serial mechanisms. The distance ratios defined in Eq. (46) for (a) and (b) must be the same.

\subsubsection{Inequality conditions for serial mechanisms}

Suppose $l_{i j}$ is the line of action of $\mathbf{w}_{i j}$ that passes through joints $J_{i}$ and $J_{j}$. First, consider the case in which $l_{i j}$ does not intersect the triangle with the other 3 joints $\left(J_{p}, J_{q}\right.$ and $\left.J_{r}\right)$ as vertices. For the locations of 5 joints shown in Fig. 6 , suppose, with no loss of generality, that $l_{12}$, the line passing through joints $J_{1}$ and $J_{2}$, does not intersect triangle $J_{3} J_{4} J_{5}$. We show that, for this case, if $\mathrm{C}$ is achieved passively at the configuration, the twist $\mathbf{t}_{12}=\mathbf{C w}_{12}$ must be centered inside triangle $J_{3} J_{4} J_{5}$. 


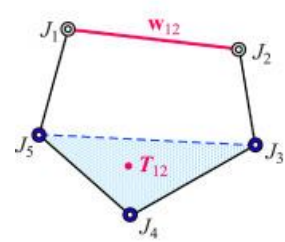

(a)

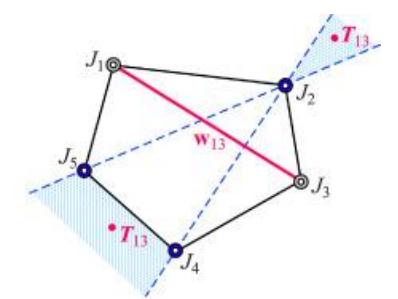

(b)

Fig. 6. Geometric interpretation on the inequality conditions. (a) Twist center $T_{12}$ is located in the triangle formed by joints $J_{3}, J_{4}$ and $J_{5}$. (b) Twist center $T_{13}$ is located outside the triangle formed by $J_{3}, J_{4}$ and $J_{5}$ and in the shaded area.

Because Eq. (14) holds for $\mathbf{C}$ with each $c_{i} \geq 0$, substituting Eqs. (12) and (14)into $\mathbf{t}_{12}=\mathbf{C w}_{12}$ yields:

$$
(46) \mathbf{t}_{12}=c_{3}\left(\mathbf{t}_{3}^{T} \mathbf{w}_{12}\right) \mathbf{t}_{3}+c_{4}\left(\mathbf{t}_{4}^{T} \mathbf{w}_{12}\right) \mathbf{t}_{4}+c_{5}\left(\mathbf{t}_{5}^{T} \mathbf{w}_{12}\right) \mathbf{t}_{5} \cdot
$$

Since the line of action of $\mathbf{w}_{12}$ does not intersect triangle $J_{3} J_{4} J_{5}$, the three vertices are on the same side of $l_{12}$. Thus, $\left(\left(\mathbf{t}_{3}^{T} \mathbf{w}_{12}\right)\right.$,), $\left(\mathbf{t}_{4}^{T} \mathbf{w}_{12}\right)$ and $\left(\mathbf{t}_{5}^{T} \mathbf{w}_{12}\right)$ must have the same sign. Because $c_{i} \geq 0$ for passive realization, the coefficients $c_{i}\left(\mathbf{t}_{i}^{T} \mathbf{w}_{12}\right)>0$, or $c_{i}\left(\mathbf{t}_{i}^{T} \mathbf{w}_{12}\right)<0(i=3,4,5)$. Because twist $\mathbf{t}_{12}$ in Eq. (47) is a positive (or negative) combination of the other three unit twists, $T_{12}$ must be located inside triangle $J_{3} J_{4} J_{5}$.

Conversely, if $T_{12}$ is located inside triangle $J_{3} J_{4} J_{5}$, then the three coefficients of $\mathbf{t}_{i}$ ( $i=$ 3,4,5) in (47), $\left(\mathbf{t}_{3}^{T} \mathbf{w}_{12}\right),,\left(\mathbf{t}_{4}^{T} \mathbf{w}_{12}\right)$ and $\left(\mathbf{t}_{5}^{T} \mathbf{w}_{12}\right)$,, must have the same sign, which indicates $c_{3}, c_{4}$ and $c_{5}$ have the same sign. We show that $c_{3}, c_{4}$ and $c_{5}$ cannot be negative.

Because $\mathbf{C}$ is a symmetric PSD matrix,

$\mathbf{w}_{12}^{T} \mathbf{C w}_{12}=c_{3}\left(\mathbf{t}_{3}^{T} \mathbf{w}_{12}\right)^{2}+c_{4}\left(\mathbf{t}_{4}^{T} \mathbf{w}_{12}\right)^{2}+c_{5}\left(\mathbf{t}_{5}^{T} \mathbf{w}_{12}\right)^{2} \geq 0$.

Therefore each $c_{i}$ must be non-negative. Thus, for this case, the inequality condition is equivalently represented by the location of $T_{12}$, which is demonstrated in Fig. 6 a.

Next, consider the case in which the axis of $\mathbf{w}_{i j}$ intersects triangle $J_{p} J_{r} J_{s}$. Here assume, without loss of generality, that the axis of $\mathbf{w}_{13}$ intersects triangle $J_{2} J_{4} J_{5}$ (as shown in Fig. 6 b). If the compliance $\mathbf{C}$ is passively achieved at the configuration shown, each coefficient $c_{i} \geq 0$ in Eq. (14). Then, similar to Eq. (47), we have:

$$
(47) \mathbf{t}_{13}=c_{2}\left(\mathbf{t}_{2}^{T} \mathbf{w}_{13}\right) \mathbf{t}_{2}+c_{4}\left(\mathbf{t}_{4}^{T} \mathbf{w}_{13}\right) \mathbf{t}_{4}+c_{5}\left(\mathbf{t}_{5}^{T} \mathbf{w}_{13}\right) \mathbf{t}_{5} .
$$

Since $J_{2}, J_{4}$ and $J_{5}$ are not all on the same side of $\mathbf{w}_{13}, \mathbf{t}_{2}^{T} \mathbf{w}_{13}, \mathbf{t}_{4}^{T} \mathbf{w}_{13}$ and $\mathbf{t}_{5}^{T} \mathbf{w}_{13}$ do not all have the same sign. Here, suppose that only $\mathbf{t}_{4}^{T} \mathbf{w}_{13}$ and $\mathbf{t}_{5}^{T} \mathbf{w}_{13}$ have the same sign. Thus, the center of $\mathbf{t}_{13}, T_{13}$, must be outside the triangle formed by $J_{2} J_{4} J_{5}$ and bounded by the two lines $J_{2} J_{4}$ and $J_{2} J_{5}$ (in the shaded area in Fig. $6 \mathrm{~b}$ ). On the other hand, if point $T_{13}$ is inside the shaded area, the coefficients of $\mathbf{t}_{4}$ and $\mathbf{t}_{5}$ in (48), $c_{4}\left(\mathbf{t}_{4}^{T} \mathbf{w}_{13}\right)$ and $c_{5}\left(\mathbf{t}_{5}^{T} \mathbf{w}_{13}\right)$, , must have the opposite sign of the coefficient of $\mathbf{t}_{2}, c_{2}\left(\mathbf{t}_{2}^{T} \mathbf{w}_{13}\right)$. Since $\left(\mathbf{t}_{2}^{T} \mathbf{w}_{13}\right)$ has a sign opposite to that of $\left(\mathbf{t}_{4}^{T} \mathbf{w}_{13}\right)$ and $\left(\mathbf{t}_{5}^{T} \mathbf{w}_{13}\right), c_{2}, c_{4}$ and $c_{5}$ have the same sign. Because $\mathbf{C}$ is a symmetric PSD matrix, by the same process used for the first case, $c_{2}, c_{4}$ and $c_{5}$ must be non-negative.

For a serial mechanism having five joints among which no three are collinear, the realization conditions can be equivalently interpreted geometrically as:

\section{Proposition 3}

A given compliance matrix $\mathbf{C}$ can be passively realized at a configuration of a 5-joint serial mechanism if and only if the following conditions hold: 
(i) The distance ratios for different sets of joints described in Fig. $5 a$ and $\underline{5} b$ are the same (satisfy Eq. (46));

(ii) A wrench whose axis passes through any 2 joints, (a) if not intersecting the triangle with the vertices being the other three joints, when multiplied by $\mathbf{C}$, yields a twist centered in the shaded triangle described in Fig. 6a; (b) if intersecting the triangle, when multiplied by $\mathbf{C}$, yields a twist centered in the shaded area described in Fig. $6 b$.

Note that condition (i) in Proposition 3 is equivalent to the equality condition (20) which ensures the decomposition of the desired compliance $\mathbf{C}$ into the form of (14). Condition (ii) for any two joints $J_{i}$ and $J_{j}$ ensures that the joint compliances for the other three joints are non-negative, and ensures that three inequality conditions in Eq. (21) are satisfied. Thus, satisfaction of condition (ii) for two sets of different joints (e.g., $\left\{J_{1}, J_{2}\right\}$ and $\left\{J_{3}, J_{4}\right\}$, or $\left\{J_{1}, J_{3}\right\}$ and $\left\{J_{2}, J_{5}\right\}$ ) guarantees all coefficients $c_{i}^{\prime}$ s in Eq. (14) are non-negative, which ensures the passive realization of $\mathrm{C}$ with the $5 \mathrm{~J}$ serial mechanism.

\subsection{Geometric significance of the realization conditions for parallel mechanisms}

By duality, the results presented in Section 4.2 for 5-joint serial mechanisms can be modified and applied to 5spring parallel mechanisms.

Suppose that $\mathbf{w}_{i}(i=1, \ldots, 5)$ are spring wrenches of a 5 -spring parallel mechanism and suppose that wrenches $\mathbf{w}_{i}$ and $\mathbf{w}_{j}$ intersect at $T_{i j}$. Then the unit twist centered at $T_{i j}, \boldsymbol{t}_{\mathrm{ij}}$, must be reciprocal to both $\mathbf{w}_{i}$ and $\mathbf{w}_{j}$. Denote:

$\mathbf{w}_{i j}=\mathbf{K t}_{i j} \cdot$

Then, $\boldsymbol{t}_{\mathrm{ij}}$ and $\mathbf{w}_{i j}$ are solely determined by the five spring wrenches and the desired stiffness $\mathbf{K}$. The realization conditions require that one equality condition in the form of $\underline{(23)}$ is satisfied and five inequality conditions in the form of $\underline{24})$ are satisfied. Below, the geometric significance of each of these conditions is identified.

\subsubsection{Equality condition for parallel mechanisms}

Using the results presented in Section 4.1, the equality condition $\underline{(23)}$ can be written as:

$\frac{\mathbf{t}_{34}^{T} \mathbf{w}_{12}}{\left(\mathbf{t}_{c}^{T} \mathbf{w}_{12}\right)\left(\mathbf{t}_{12}^{T} \mathbf{w}_{5}\right)\left(\mathbf{t}_{34}^{T} \mathbf{w}_{5}\right)}=\frac{\mathbf{t}_{14}^{T} \mathbf{w}_{23}}{\left(\mathbf{t}_{c}^{T} \mathbf{w}_{23}\right)\left(\mathbf{t}_{23}^{T} \mathbf{w}_{5}\right)\left(\mathbf{t}_{14}^{T} \mathbf{w}_{5}\right)}$

Since the screw product of a unit twist and a unit wrench indicates the distance from the twist center to the wrench axis, normalizing the wrenches and twists yields

$$
\text { (48) } \frac{d_{12}^{34}}{d_{12}^{c} d_{5}^{12} d_{5}^{34}}=\frac{d_{23}^{14}}{d_{23}^{c} d_{5}^{23} d_{5}^{14}},
$$

where $d_{p q}^{i j}$ is the distance from the twist center of $\boldsymbol{t}_{\mathrm{ij}}$ to the wrench axis of $\mathbf{w}_{\mathrm{pq}}$. The geometric meaning of each distance $d_{p q}^{i j}$ in Eq. (49) is illustrated in Figs. 7a and $\underline{7 b}$. The ratios of the distances in Eq. (49) in the two figures must be equal. Thus, the equality realization condition is expressed in terms of the mechanism geometry and stiffness properties.

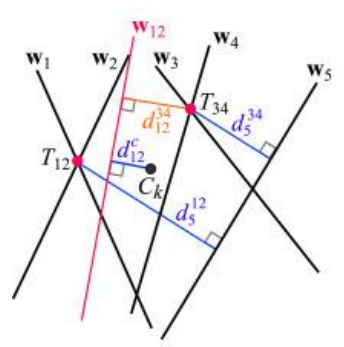

(a)

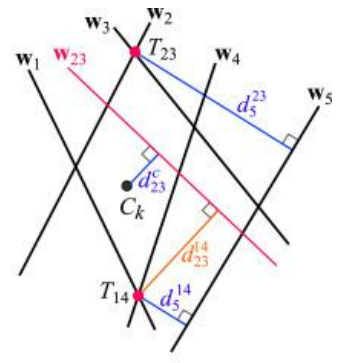

(b) 
Fig. 7. Geometric interpretation on the equality conditions for parallel mechanisms. The distance ratios defined in Eq. (49) for (a) and (b) must be the same.

\subsubsection{Inequality conditions for parallel mechanisms}

Suppose a 5-spring parallel mechanism has spring wrenches $\mathbf{w}_{i}(i=1,2, \ldots, 5)$ among which no three are concurrent or parallel to each other. Dual to Eq. (14), a stiffness $\mathrm{K}$ can be passively achieved by the mechanism if and only if $\mathbf{K}$ can be expressed as:

$$
\text { (49) } \mathbf{K}=k_{1} \mathbf{w}_{1} \mathbf{w}_{1}^{T}+k_{2} \mathbf{w}_{2} \mathbf{w}_{2}^{T}+k_{3} \mathbf{w}_{3} \mathbf{w}_{3}^{T}+k_{4} \mathbf{w}_{4} \mathbf{w}_{4}^{T}+k_{5} \mathbf{w}_{5} \mathbf{w}_{5}^{T},
$$

with each $k_{i} \geq 0$.

Suppose that $\boldsymbol{t}_{\mathrm{ij}}$ is the unit twist centered at $T_{i j}$, the intersection of wrenches $\mathbf{w}_{i}$ and $\mathbf{w}_{j}$, and that $\mathbf{w}_{i j}$ is the wrench resulting from $t_{\mathrm{ij}}$ imposed on $\mathbf{K}$, i.e.,

$\mathbf{w}_{i j}=\mathbf{K t}_{i j} \cdot$

Using a procedure equivalent to that for serial mechanisms, suppose, without loss of generality, $(i, j)=$ $(1,2)$,, and first consider the case in which $T_{12}$ is located in the triangle formed by the other three wrenches $\mathbf{w}_{3}, \mathbf{w}_{4}, \mathbf{w}_{5}$ (as shown in Fig. 8a). For this case, it is shown that the line of action of $\mathbf{w}_{12}$ does not intersect the triangle.

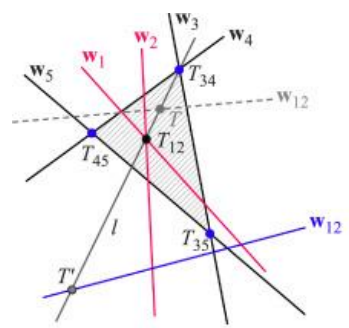

(a)

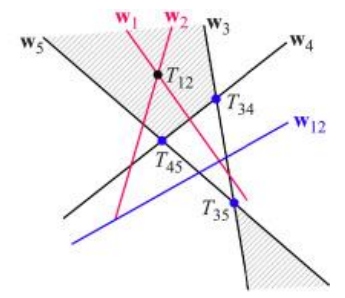

(b)

Fig. 8. Geometric interpretation of the inequality conditions. A twist centered at $T_{12}$, (a) if located in the triangle formed by wrench axes $\mathbf{w}_{3}, \mathbf{w}_{4}$ and $\mathbf{w}_{5}$, when multiplied by $\mathbf{K}$, yields a wrench $\mathbf{w}_{12}$ that does not intersect the triangle; (b) if located outside the triangle, when multiplied by $\mathbf{K}$, yields a wrench $\mathbf{W}_{12}$ that intersects the triangle without crossing the shaded area.

In fact,

$$
(50) \mathbf{w}_{12}=\mathbf{K t}_{12}=k_{3}\left(\mathbf{w}_{3}^{T} \mathbf{t}_{12}\right) \mathbf{w}_{3}+k_{4}\left(\mathbf{w}_{4}^{T} \mathbf{t}_{12}\right) \mathbf{w}_{4}+k_{5}\left(\mathbf{w}_{5}^{T} \mathbf{t}_{12}\right) \mathbf{w}_{5} .
$$

Then,

$$
\text { (51) } \mathbf{t}_{34}^{T} \mathbf{K t}_{12}=k_{5}\left(\mathbf{w}_{5}^{T} \mathbf{t}_{12}\right)\left(\mathbf{w}_{5}^{T} \mathbf{t}_{34}\right) \text {. }
$$

Since $T_{12}$ is in the triangle, $T_{12}$ and $T_{34}$ are on the same side of $\mathbf{w}_{5}$. Thus, $\mathbf{w}_{5}^{T} \mathbf{t}_{12}$ and $\mathbf{w}_{5}^{T} \mathbf{t}_{34}$ have the same sign, which together with Eq. (52) indicates $\mathbf{t}_{34}^{T} \mathbf{K t}_{12}>0$. Similarly, $\mathbf{t}_{35}^{T} \mathbf{K t}_{12}>0$,, $\mathbf{t}_{45}^{T} \mathbf{K t}_{12}>0$. Summarizing,

$$
(52) \mathbf{t}_{34}^{T} \mathbf{K t}_{12}>0, \mathbf{t}_{35}^{T} \mathbf{K t}_{12}>0, \mathbf{t}_{45}^{T} \mathbf{K t}_{12}>0 \text {.. }
$$

If $\mathbf{w}_{12}$ intersects the triangle, it must intersect one of three line segments: $T_{12} T_{34}, T_{12} T_{35}$, or $T_{12} T_{45}$. Suppose that $\mathbf{w}_{12}$ intersects segment $T_{12} T_{34}$ at $T$ (as shown by the dashed line in Fig. 8a), and $\mathbf{t}$ is the unit twist centered at $T$. Then, since $T$ is located between $T_{12}$ and $T_{34}$, twist $t$ can be expressed as a positive combination of the two unit twists $\mathbf{t}_{12}$ and $\mathbf{t}_{34}$ : 
$\mathbf{t}=\alpha \mathbf{t}_{12}+\beta \mathbf{t}_{34 \prime \prime}$

where $\alpha>0$ and $\beta>0$. Then,

$\mathbf{t}^{T} \mathbf{w}_{12}=\left(\alpha \mathbf{t}_{12}+\beta \mathbf{t}_{34}\right)^{T} \mathbf{K} \mathbf{t}_{12}=\alpha \mathbf{t}_{12}^{T} \mathbf{K t}_{12}+\beta \mathbf{t}_{34}^{T} \mathbf{K t}_{12}=0$.

Since $\mathbf{t}_{12}^{T} \mathbf{K t}_{12} \geq 0, \alpha>0$ and $\beta>0$,

$(53) \mathbf{t}_{34}^{T} \mathbf{K t}_{12}<0$,

which conflicts with the first inequality in (53). Therefore, $\mathbf{w}_{12}$ cannot intersect segment $T_{12} T_{34}$. By the same reasoning, $\mathbf{w}_{12}$ cannot intersect segment $T_{12} T_{35}$ or segment $T_{12} T_{45}$. Thus, $\mathbf{w}_{12}$ cannot intersect triangle $J_{3} J_{4} J_{5}$.

Conversely, if the line of action of $\mathbf{w}_{12}$ does not intersect the triangle, then, the three

vertices $T_{34}, T_{35}$ and $T_{45}$ of the triangle must be on the same side of $\mathbf{w}_{12}$, and

thus $\mathrm{t}_{34}^{T} \mathrm{w}_{12}, \mathrm{t}_{35}^{T} \mathrm{~W}_{12}$ and $\mathbf{t}_{45}^{T} \mathbf{w}_{12}$ must have the same sign. Below, we show these three quantities must be positive. To prove this, we only need to show that $\mathrm{t}_{34}^{T} \mathrm{w}_{12}>0$,.

Consider the line / passing through $T_{12}$ and $T_{34}$ which intersects $\mathbf{w}_{12}$ at $T^{\prime}$ (as shown Fig. 8a). Since $T^{\prime}$ is outside the triangle, it must be outside the segment $T_{12} T_{34}$. Then, the unit twist $\mathbf{t}^{\prime}$ centered at $T^{\prime}$ can be expressed as

$\mathbf{t}^{\prime}=\alpha^{\prime} \mathbf{t}_{12}+\beta^{\prime} \mathbf{t}_{34 \prime \prime}$

where the two scalars $\alpha^{\prime}$ and $\beta^{\prime}$ have opposite signs, i.e., $\alpha^{\prime} \beta^{\prime}<0$. Since $\mathbf{t}^{\prime}$ and $\mathbf{w}_{12}$ are reciprocal,

$\mathbf{t}^{\prime T} \mathbf{w}_{12}=\left(\alpha^{\prime} \mathbf{t}_{12}+\beta^{\prime} \mathbf{t}_{34}\right)^{T} \mathbf{K} \mathbf{t}_{12}=\alpha^{\prime} \mathbf{t}_{12}^{T} \mathbf{K t}_{12}+\beta^{\prime} \mathbf{t}_{34}^{T} \mathbf{K t}_{12}=0$.

Thus, $\mathbf{t}_{12}^{T} \mathbf{K t}_{12}$ and $\mathbf{t}_{34}^{T} \mathbf{K t}_{12}$ must have the same sign. Because $\mathbf{t}_{12}^{T} \mathbf{K t}_{12}>0$, then $\mathbf{t}_{34}^{T} \mathbf{K t}_{12}>0$, satisfying the first inequality of (53). With similar procedures, the three inequalities in (53) are each satisfied, which implies that $k_{3}, k_{4}$ and $k_{5}$ in Eq. (51) are non-negative. Summarizing, coefficients $k_{3}, k_{4}$ and $k_{5}$ in Eq. (51) are nonnegative, if and only if $\mathbf{w}_{12}$ does not intersect the shaded triangle formed by wrenches $\mathbf{w}_{3}, \mathbf{w}_{4}$, and $\mathbf{w}_{5}$ as illustrated in Fig. 8a.

Second, consider the case in which $T_{12}$ is located outside the triangle formed by wrenches $\mathbf{w}_{3}, \mathbf{w}_{4}$, and $\mathbf{w}_{5}$. Using a process similar to that used for the serial case, it can be proved that, for this case, the line of action of $\mathbf{w}_{12}$ must intersect the triangle without crossing the shaded area described in Fig. 8 $\mathrm{b}$.

For a parallel mechanism having five springs among which no three are concurrent or parallel to each other, the realization conditions can be equivalently interpreted geometrically as:

\section{Proposition 4}

A stiffness matrix $\mathbf{K}$ can be passively realized with a 5-spring parallel mechanism if and only if the following conditions hold:

(i) The distance ratios for different sets of wrenches described in Fig. 7a and $7 b$ are the same (satisfy Eq. (49));

(ii)A twist centered at the intersection of 2 spring axes, (a) if located within the triangle enclosed by the other 3 springs, when multiplied by $\mathbf{K}$, yields a wrench that does not intersect the triangle; (b) if located outside the triangle, when multiplied by $\mathbf{K}$, yields a wrench that intersects the triangle without crossing the shaded area described in Fig. 8 b.

Dual to the serial case, condition (i) in Proposition 4 ensures the decomposition of $\mathbf{K}$ into the form of Eq. (50). Condition (ii) of Proposition 4 for any two springs ensures that the spring stiffnesses for the other three springs 
are non-negative. Thus, satisfaction of condition (ii) for two sets of different springs (e.g., $\left\{\mathbf{w}_{1}, \mathbf{w}_{2}\right\}$ and $\left\{\mathbf{w}_{3}, \mathbf{w}_{4}\right\}$, or $\left\{\mathbf{w}_{1}, \mathbf{w}_{3}\right\}$ and $\left.\left\{\mathbf{w}_{2}, \mathbf{w}_{4}\right\}\right)$ guarantees the passive realization of $\mathbf{K}$ with the 5-spring parallel mechanism.

\subsection{The compliance center and the mechanism configuration}

For any given planar compliant behavior, there is a particular point at which both the compliance matrix and stiffness matrix can be described in a diagonal form. This unique point is defined to be the center of compliance/stiffness. It was shown [6], [30] that, if a compliant behavior is passively achieved with a mechanism having 3 or 4 elastic components, then the center must be located within an area enclosed by the locations of these components. These results can be extended to mechanisms having five elastic components.

First consider a serial $5 \mathrm{~J}$ mechanism. Let $l_{i j}$ be the line passing through $J_{i}$ and $J_{j}$, and $\mathbf{w}_{i j}$ be the unit wrench associated with $l_{i j}$. Suppose, with no loss of generality, that $l_{12}$ does not intersect the triangle with vertices at the other joints $J_{3}, J_{4}$ and $J_{5}$. Then by realization inequality condition (i) of Proposition $3, T_{12}$, the center of twist $T_{12}=\mathrm{Cw}_{12}$, must be inside triangle $J_{3} J_{4} J_{5}$. Since the compliance center and $T_{12}$ must be on the same side of $l_{12}$ [30], $C_{c}$ must be on the same side of $l_{12}$ as the triangle (the shaded area as illustrated in Fig. 9a). Applying the same process to all lines $l_{i j}$ that have the other three joints on the same side, the center of compliance must be within the convex hull formed by the five joints as demonstrated in Fig. 9 b.

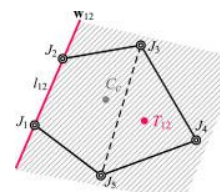

(a)

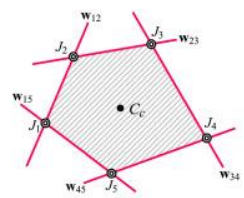

(b)

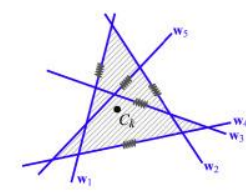

(c)

Fig. 9. Locus of compliance centers $C_{c}$. (a) When considering only two joints, $J_{1}$ and $J_{2}, C_{c}$ is on the same side of line $l_{12}$ as triangle $J_{3} J_{4} J_{5}$. (b) When considering all joints, $C_{c}$ must be within the convex hull formed by the five joints. (c) For a 5 -spring parallel mechanism, the stiffness center $C_{k}$ must be the within the union of triangles formed by any three spring axes.

Similarly, it can be shown that, if a stiffness is passively achieved by a 5-spring parallel mechanism, the stiffness center must be inside the union of triangles formed by any 3 spring axes as illustrated in Fig. 9c. Note that for a 5 -spring parallel mechanism, the locus area of stiffness centers $C_{k}$ is not convex.

\section{Compliance synthesis}

In this section, synthesis procedures for both types of mechanisms are developed using the conditions on the mechanism geometry developed in Section 4. In each procedure, the center of the elastic behavior is used.

\subsection{Synthesis with a 5-joint serial mechanism}

Suppose $\mathbf{C}$ is a $3 \times 3$ compliance matrix having the form:

$\mathbf{C}=\left[\begin{array}{cc}\mathbf{A} & \mathbf{b} \\ \mathbf{b}^{T} & c_{33}\end{array}\right]$

the compliance center $C_{C}$ is determined by:

$$
\text { (54) } \mathbf{r}_{c}=\frac{1}{c_{33}} \mathbf{S b},
$$

where $\mathbf{S}$ is the $2 \times 2$ matrix defined in Eq. (3). The unit twist $\mathbf{t}_{c}$ associated with $C_{c}$ is calculated using Eq. (1).

The procedure for a 5 -joint serial mechanism realization of compliance $\mathbf{C}$ presented below identifies the locations of the five joints and the corresponding joint compliances. Selections used in the procedure are illustrated in Fig. 10. 
1. Select arbitrarily a line $l_{12}$ and obtain the corresponding unit wrench $\mathbf{w}_{12}$. Two joints $J_{1}$ and $J_{2}$ will lie on line $l_{12}$. The associated twist is calculated:

$$
(55) \mathbf{t}_{12}=\mathbf{C w}_{12} \cdot
$$

The unit twist associated with $\mathbf{t}_{12}, \hat{\mathbf{t}}_{12}$, is obtained and the center of $\hat{\mathbf{t}}_{12}, T_{12}$, is calculated using Eq. (2).

2. Select arbitrarily a line $l_{34}$ (represented by unit wrench $\mathbf{w}_{34}$ ). Two joints $J_{3}$ and $J_{4}$ will lie on line $l_{34}$.

3. Select the location of joint $J_{5}$. To satisfy condition (ii) of Proposition 3 , this point must be located on the same side of $l_{34}$ as $T_{12}$. The unit twist centered at $J_{5}, \mathbf{t}_{5}$, is obtained using Eq. (1).

4. Select a line $l_{23}$ (represented by wrench $\mathbf{w}_{23}$ ) that intersects $l_{12}$ and $l_{34}$ to obtain joint locations $J_{2}$ and $J_{3}$. This line must be judiciously chosen such that $T_{34}$ satisfies condition (ii) of Proposition 3 . The twist associated with $\mathbf{w}_{23}$ is calculated:

(57) $\mathbf{t}_{23}=\mathrm{Cw}_{23}$.

The unit twist associated with $\mathbf{t}_{23}, \hat{\mathbf{t}}_{23}$, is obtained, and the center of $\mathbf{t}_{23}, T_{23}$, is calculated using Eq. (2). The distance ratio used in the equality condition corresponding to the selections of $\mathbf{w}_{12}, \mathbf{w}_{34}, \mathbf{w}_{23}$ and $J_{5}$ is calculated:

(58) $\gamma_{S}=\frac{\left(\mathbf{w}_{12}^{T} \mathbf{t}_{c}\right)\left(\mathbf{w}_{34}^{T} \hat{\mathbf{t}}_{12}\right)\left(\mathbf{w}_{22}^{T} \mathbf{t}_{5}\right)}{\left(\mathbf{w}_{12}^{T} \mathbf{t}_{5}\right)\left(\mathbf{w}_{34}^{T} \mathbf{t}_{5}\right)\left(\mathbf{w}_{23}^{T} \mathbf{t}_{c}\right)}$.

Note that in calculation of the ratio $\gamma_{s}$ in Eq. (58), normalization of $\mathbf{w}_{12}, \mathbf{w}_{34}$ and $\mathbf{w}_{23}$ to unit wrenches is not necessary since they appear in both the numerator and denominator.

5. Choose a line $\mathbf{w}_{14}$ such that the ratio of the distances from the line to $T_{23}$ and $J_{5}$ is equal to $\gamma_{s}$. This can be accomplished by the following steps.

(a) On line $l_{p}$ passing through $T_{23}$ and $J_{5}$, choose point $P$ such that the ratio of the distances from $T_{23}$ to $P$ and from $J_{5}$ to $P$ is $\gamma_{s}$. This point $\left(x_{p}, y_{p}\right)$ can be determined by the two equations:

(59) $\frac{\left\|\mathbf{t}_{p}-\hat{\mathbf{t}}_{23}\right\|}{\left\|\mathbf{t}_{p}-\mathbf{t}_{5}\right\|}=\left|\gamma_{s}\right|, \mathbf{t}_{p} \cdot\left(\hat{\mathbf{t}}_{23} \times \mathbf{t}_{5}\right)=0$,

where $\mathbf{t}_{p}=\left[y_{p},-x_{p}, 1\right]^{T}$ is the unit twist located at $P$, and $\hat{\mathbf{t}}_{23}$ and $\mathbf{t}_{5}$ are the unit twists at $T_{23}$ and $J_{5}$, respectively. Note that the set of equations in (59) yields two solutions on line $l_{p}$ : one is inside line segment $J_{5} T_{23}$, and the other is outside. The selection of point $P$ for the realization depends on the sign of $\gamma_{s}$ : if $\gamma_{s}>0$, the solution outside segment $J_{5} T_{23}$ is selected; if $\gamma_{s}<0$, the solution inside segment $J_{5} T_{23}$ is selected. Thus $P$ is uniquely determined.

(b) Any line passing through point $P$ satisfies the ratio condition. Judiciously select a line $l_{14}$ passing through $P$ (denoted as $\mathbf{w}_{14}$ ) that intersects $\mathbf{w}_{12}$ and $\mathbf{w}_{34}$ to obtain joint locations $J_{1}$ and $J_{4}$. In the selection of this line, condition (ii) of Proposition 3 for both $T_{12}$ and $T_{34}$ must be satisfied.

6. Calculate the joint compliances using Eq. (25).

With this final step, the five joint locations and compliances in the serial mechanism are identified. 


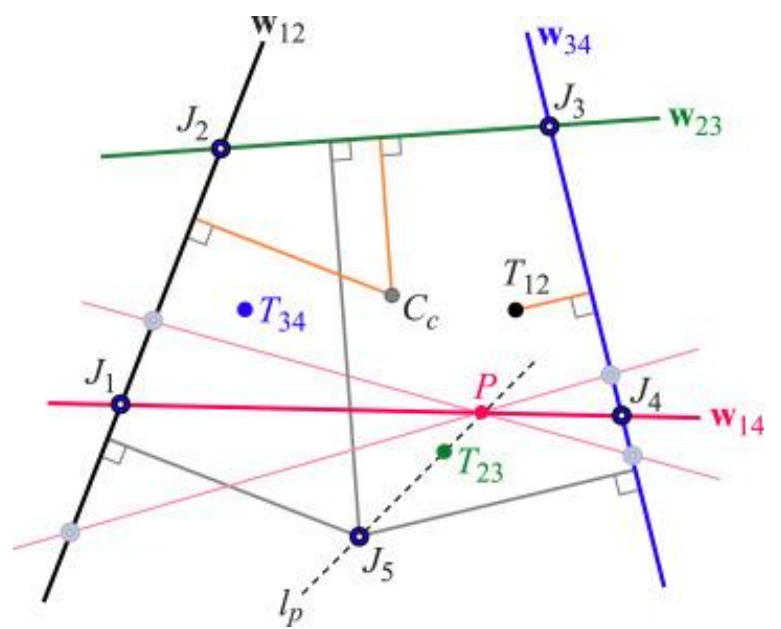

Fig. 10. Compliance synthesis with a 5-joint serial mechanism using geometric constraints.

\subsection{Synthesis with a 5-spring parallel mechanism}

Suppose $\mathbf{K}$ is a given $3 \times 3$ stiffness matrix having the form:

$\mathbf{K}=\left[\begin{array}{cc}\mathbf{D} & \mathbf{v} \\ \mathbf{v}^{T} & k_{33}\end{array}\right]$

the center of stiffness is calculated by:

(60) $\mathbf{r}_{c}=-\mathbf{S D}^{-1} \mathbf{v}$

where $\mathbf{S}$ is the $2 \times 2$ matrix defined in Eq. (3). The unit twist $\mathbf{t}_{c}$ associated with the stiffness center $C_{k}$ is calculated using Eq. (1).

Below, the synthesis procedure for stiffness realization with a 5-spring parallel mechanism is presented. Selections used in the process are shown in Fig. 11.

1. Select arbitrarily a point $T_{12}$ and calculate the unit twist $t_{12}$ centered at $T_{12}$ using Eq. (1). Two spring axes $\mathbf{w}_{1}$ and $\mathbf{w}_{2}$ of the mechanism will intersect at this point. The wrench associated with $\mathbf{t}_{12}$ is calculated:

(61) $\mathbf{w}_{12}=\mathbf{K t}_{12}$.

The unit wrench associated with $\mathbf{w}_{12}, \widehat{\mathbf{w}}_{12}$, is obtained and the line of action of $\mathbf{w}_{12}$ is interpreted geometrically using Eq. (4).

2. Select another point $T_{34}$ arbitrarily. Calculate the unit twist $\mathbf{t}_{34}$ associated with $T_{34}$. The wrench corresponding to $\mathbf{t}_{34}$ is calculated:

(62) $\mathbf{w}_{34}=\mathbf{K t}_{34}$.

With the unit wrench associated with $\mathbf{w}_{34}$, the line of action of $\mathbf{w}_{34}$ is again interpreted geometrically using Eq. (4). Two spring axes $\mathbf{w}_{3}$ and $\mathbf{w}_{4}$ will intersect at $T_{34}$.

3. Select line $l_{5}$, which will be the line of action of spring $\mathbf{w}_{5}$. To satisfy condition (ii) of Proposition 4 , this line must be selected such that points $T_{12}$ and $T_{34}$ are on the same side of $l_{5}$. The unit spring wrench $\mathbf{w}_{5}$ is obtained using Eq. (4).

4. 
Judiciously select a point $T_{23}$, at which spring axes $\mathbf{w}_{2}$ and $\mathbf{w}_{3}$ will meet. This point, together with points $T_{12}$ and $T_{34}$, determines wrenches $\mathbf{w}_{2}$ and $\mathbf{w}_{3}$. In selecting this point, condition (ii) of Proposition 4 must be satisfied. The wrench corresponding to the twist at $T_{23}$ is:

(63) $\mathbf{w}_{23}=\mathbf{K t}_{23} \cdot$

Calculate the distance ratio used in the equality condition corresponding to the selections of $T_{12}, T_{34}, T_{23}$ and $\mathbf{w}_{5}$ :

(64) $\gamma_{p}=\frac{\left(\mathbf{t}_{34}^{T} \mathbf{w}_{12}\right)\left(\mathbf{t}_{c}^{T} \mathbf{w}_{23}\right)\left(\mathbf{t}_{23}^{T} \mathbf{w}_{5}\right)}{\left(\mathbf{t}_{c}^{T} \mathbf{w}_{12}\right)\left(\mathbf{t}_{12}^{T} \mathbf{w}_{5}\right)\left(\mathbf{t}_{34}^{T} \mathbf{w}_{5}\right)}$

5. Select a point $T_{14}$ such that the ratio of the distances from the point to lines of $\mathbf{w}_{23}$ and $\mathbf{w}_{5}$ is equal to $\gamma_{p}$, i.e., (65) $\frac{\mathbf{t}_{14}^{T} \mathbf{w}_{23}}{\mathbf{t}_{14}^{T} \mathbf{w}_{5}}=\gamma_{p}$

This can be accomplished by the following steps.

(a) For the given $\mathbf{w}_{23}$ and $\mathbf{w}_{5}$, consider the equation defined by:

(66) $\mathbf{t}^{T} \mathbf{w}_{23}=\gamma_{p}\left(\mathbf{t}^{T} \mathbf{w}_{5}\right)$,

where $\mathbf{t}$ is a unit twist defined by $\mathbf{t}=[y,-x, 1]^{T}$. Then Eq. (66) defines a line $l_{t}$ on which any point satisfies the equality condition.

(b) Judiciously choose one point $T_{14}$ on line $l_{t}$ such that condition (ii) of Proposition 4 for both $\mathbf{w}_{12}$ and $\mathbf{w}_{34}$ are satisfied. The remaining four spring axes $\left(\mathbf{w}_{i}, i=1,2,3,4\right)$ are determined by the four lines passing through points $\left(T_{12}, T_{14}\right),\left(T_{12}, T_{23}\right),\left(T_{23}, T_{34}\right)$, and $\left(T_{14}, T_{34}\right)$ as illustrated in Fig. 11.

6. Calculate the stiffness for each spring using Eq. (26).

With this final step, the five spring axes and their stiffnesses in the parallel mechanism are identified.

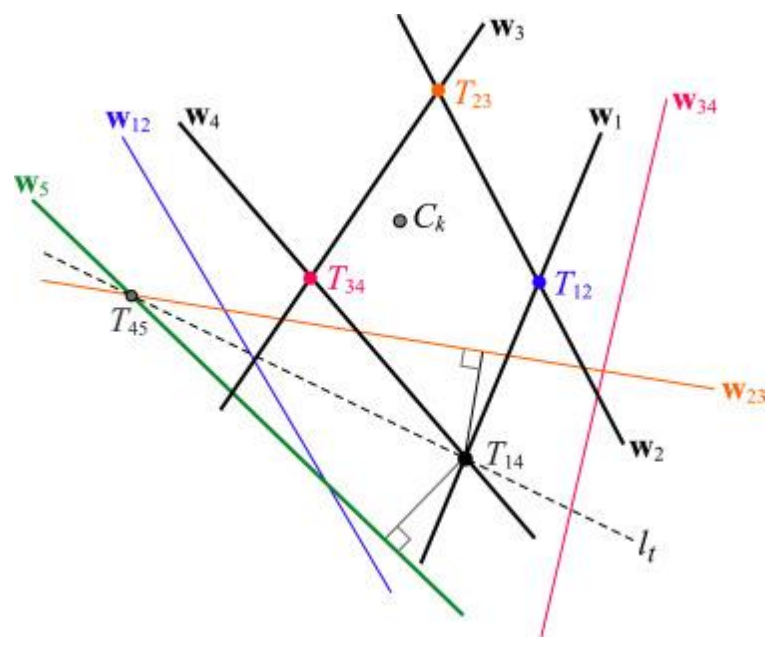

Fig. 11. Compliance synthesis with a 5-spring parallel mechanism based on geometry.

\subsection{Discussion}

In each step of the procedures presented in Sections 5.1 and $\underline{5.2}$, a line or a point is selected. Since the elastic components (joints or springs) in a mechanism can be numbered arbitrarily, the order of these selections can be changed. 
When the mechanism configuration is determined, each joint compliance in the serial mechanism (or each spring constant in the parallel mechanism) can be calculated using any permutation in Eq.

(25) or (26) provided that all twists and wrenches involved have finite (zero) pitch. Thus, any translational twist or rotational wrench (having infinite pitch) should not be used in Eq. (25) or (26) to calculate the joint compliances or spring stiffnesses.

In application, constraints on the mechanism configuration need to be considered. For example, if it is desired that the first joint $J_{1}$ and the last joint $J_{5}$ be located at given locations (e.g., when the location of the robot base and end-effector pose are specified), the synthesis of a given compliance involves identifying the three remaining joint locations $J_{2}, J_{3}$ and $J_{4}$. The procedure presented in Section 5.1 can be modified to meet this requirement and is described below.

First, choose a line $l_{12}$ to pass through the given point $J_{1}$ and choose $J_{5}$ at the location yielding the desired endeffector pose. Then select line $l_{34}$ at a location that satisfies condition (ii) of Proposition 3 . Line $l_{23}$ can be selected as described in Step 4 to determine $J_{2}$ and $J_{3}$, and point $P$ is calculated using the method described in Step 5a. By passing through points $J_{1}$ and $P$, line $l_{14}$ is determined. The intersection of $l_{34}$ and $l_{14}$ is the location of $J_{4}$. It can be seen that, since the locations of two joints are specified, line $l_{14}$ is unique.

Note that in the compliance realization with a 5-joint mechanism, at most two joint locations can be specified arbitrarily. If three joint locations are constrained, the mechanism may not have sufficient mobility to achieve a configuration required by the realization, and thus would not be able to realize the given behavior. Once the first two joint locations are selected, the remaining joint locations must be selected such that geometric condition (ii) in Proposition 3 is satisfied. The serial elastic mechanism synthesis example in the next section considers practical constraints.

In the synthesis process, the space of possible joint locations for each joint is identified. One can select any one from the available space based on its geometry. When five joint locations are selected, the corresponding joint compliances can be determined by Eq. (25). Since the selection of each joint is not unique, if different configurations are selected in the process, a different set of joint compliances will be obtained using Eq. (25). It is guaranteed that all joint compliances calculated by Eq. (25) are positive for any configuration selected using the procedure. Also, in selecting the location of each joint, mechanism singularity (for which three joints are located on a straight line) should be avoided. This can always be accomplished since there are infinitely many options in the space of joint locations.

Note that, once the joint locations are identified, the specified compliance is realized only at that configuration. Since a $5 \mathrm{~J}$ serial mechanism has redundancy, the nullspace of the Jacobian is nonzero and thus kinematic internal motion allows the mechanism configuration to change with the endpoint pose relative to the base unchanged. The realization may not be maintained for a variation from the obtained configuration because a change of the configurations by an internal motion may violate the realization conditions.

It can be seen that to realize a given compliance using a serial mechanism with two specified joint locations, the mechanism must have at least 5 joints. This is because if the number of joints of a mechanism is less than 5 , the mechanism does not have the necessary degree of freedom to satisfy the corresponding realization conditions.

Similar to the serial case, for parallel mechanism synthesis with two spring axes being specified, the mechanism must have at least five springs in order to realization an arbitrary stiffness. For a 5-spring parallel mechanism, the first two spring wrenches can always be selected arbitrarily. In the selection of the remaining springs, the previously selected spring wrenches and geometric condition (ii) in Proposition 4 must be satisfied. Also, in selecting the spring axes, mechanism singularity (three springs intersecting at a single point or parallel to each 
other) should be avoided. This can always be accomplished since there are infinitely many options in the space of acceptable spring wrenches.

Compared to previous results [30] for 4-component mechanisms, the advantages of the 5-component mechanisms considered in the paper are that the dimension of the space of realizable compliances at a configuration is increased by one and that more geometric constraintson the mechanism can be accommodated. The disadvantage is that more components increase the complexity of the mechanism.

\section{Synthesis procedure examples}

Numerical examples for both serial and parallel mechanisms are presented to demonstrate the synthesis procedures. For serial mechanism synthesis, the procedure identifies a serial mechanism that realizes the given compliance $\mathbf{C}$ by selecting the locations of five joints and their corresponding joint compliances. For parallel mechanism synthesis, the procedure identifies a parallel mechanism that realizes the given stiffness $\mathbf{K}$ by selecting the axes of five springs and their corresponding spring constants.

In a global coordinate frame, the compliant behavior to be realized is specified by:

(67) $\mathbf{K}=\left[\begin{array}{ccc}6 \mathrm{~N} / \mathrm{m} & -4 \mathrm{~N} / \mathrm{m} & 2 \mathrm{~N} \\ -4 \mathrm{~N} / \mathrm{m} & 12 \mathrm{~N} / \mathrm{m} & 10 \mathrm{~N} \\ 2 \mathrm{~N} & 10 \mathrm{~N} & 18 \mathrm{~N} \cdot \mathrm{m}\end{array}\right], \mathbf{C}=\mathbf{K}^{-1}=\left[\begin{array}{ccc}0.58 \mathrm{~m} / \mathrm{N} & 0.46 \mathrm{~m} / \mathrm{N} & -0.32 \mathrm{~N}^{-1} \\ 0.46 \mathrm{~m} / \mathrm{N} & 0.52 \mathrm{~m} / \mathrm{N} & -0.34 \mathrm{~N}^{-1} \\ -0.32 \mathrm{~N}^{-1} & -0.34 \mathrm{~N}^{-1} & 0.28(\mathrm{~N} \cdot \mathrm{m})^{-1}\end{array}\right]$.

In the examples, the units of all wrenches and twists calculated in the synthesis process are consistent with that used in $\mathbf{K}$ and $\mathbf{C}$. For a twist, the first two components have units of length in meters [m] and the third component has units of radian. For a wrench, the first two components have units of force in Newton [N] and the third component has units of moment in $N \cdot m$. Also, all Cartesian coordinates are in meters.

Using Eq. (55) or $\underline{(60)}$, the stiffness/compliance center of this behavior is calculated to be located at $\left(\frac{17}{14},-\frac{8}{7}\right)$. The unit twist at the center is

$\mathbf{t}_{c}=\left[-\frac{8}{7},-\frac{17}{14}, 1\right]^{T}$

\subsection{Synthesis with a 5-joint serial mechanism}

In this example, it is desired that joints $J_{1}$ (the joint connected to the base of the manipulator) and $J_{5}$ (the joint connected to the manipulator end-effector) be at given locations. In the coordinate frame used to describe $\mathbf{C}$, the two joints $J_{1}$ and $J_{5}$ are required to be located at

$\mathbf{r}_{1}=[-2,-2]^{T}, \mathbf{r}_{5}=[2,-2]^{T}$.

Below, the locations of the remaining three joints are identified using the modified synthesis procedure presented in Section 5.3.

The unit twists associated with $J_{1}$ and $J_{5}$ are calculated using Eq. (1) to be:

$\mathbf{t}_{1}=[-2,2,1]^{T}, \mathbf{t}_{5}=[-2,-2,1]^{T}$.

The modified serial mechanism synthesis procedure of Section 5.3 is used to determine the other three joint locations $\left(U_{2}, J_{3}, J_{4}\right)$, and all joint compliance values. The component geometry selections used in the process are illustrated in Fig. 12. 


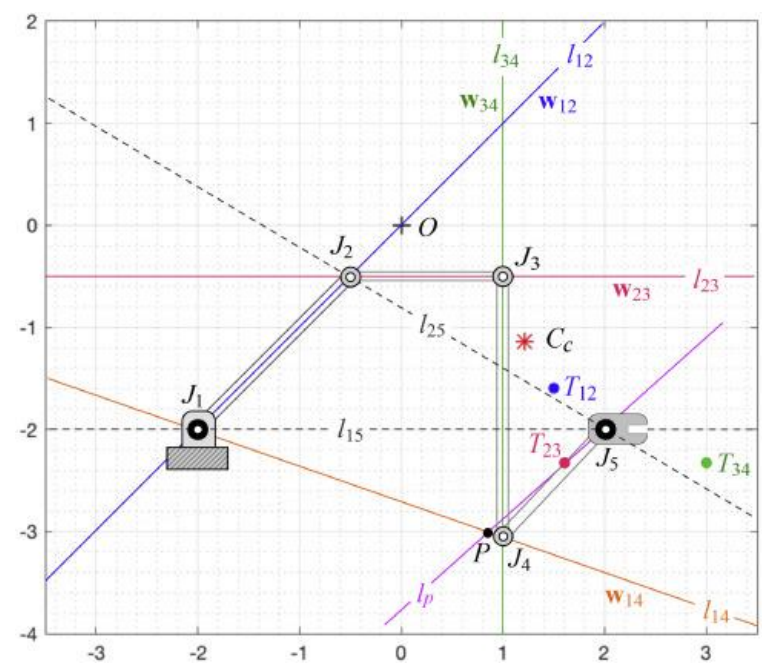

Fig. 12. Compliance synthesis with a 5 -joint mechanism with $J_{1}$ and $J_{5}$ being specified. The desired compliance is realized by choosing the remaining three joint locations and all joint compliances in the serial mechanism.

The first line $l_{12}$ is selected to pass through $J_{1}$ at $(-2,-2)$ with an arbitrary slope. Here, the slope is selected to be 1 . The unit wrench representing $l_{12}$ is:

$\mathbf{w}_{12}=\frac{\sqrt{2}}{2}[1,1,0]^{T}$.

The twist $\mathbf{t}_{12}$ corresponding to $\mathbf{w}_{12}$ is calculated to be:

$\hat{\mathbf{t}}_{12}=[-1.5758,-1.4848,1]^{T}$.

Normalizing $\mathbf{t}_{12}$ yields the unit twist:

$\hat{\mathbf{t}}_{12}=[-1.5758,-1.4848,1]^{T}$,

and the location of the center of $\mathbf{t}_{12}, T_{12}$, is calculated to be $(1.4848,-1.5758)$.

The second line $l_{34}$ is where joints $J_{3}$ and $J_{4}$ will lie. Since the location of $J_{5}$ has been specified, $l_{34}$ must be selected such that $T_{12}$ and $J_{5}$ are on the same side of $l_{34}$; otherwise $T_{12}$ will be outside of the triangle formed by $J_{3}, J_{4}$ and $J_{5}$ (which violates the inequality condition (ii) of Proposition 3 ). In this example, $l_{34}$ is chosen to pass through point $(1,0)$ and parallel to $y$-axis. The unit wrench representing $l_{34}$ is:

$\mathbf{w}_{34}=[0,1,1]^{T}$.

The associated twist $\mathbf{t}_{34}$ is:

$\mathbf{t}_{34}=\mathbf{C w}_{34}=[0.14,0.18,-0.06]^{T}$.

The twist center of $\mathbf{t}_{34}, T_{34}$, is determined to be $(3,-2.3333)$.

Select a line $l_{23}$ to meet lines $l_{12}$ and $l_{34}$ to determine the locations of $J_{2}$ and $J_{3}$. This line should be chosen such that $T_{34}$ is above line $I_{25}$ (which passes $J_{2}$ and $J_{5}$ as shown in Fig. 12) to satisfy condition (ii) of Proposition 3. Here, a line passing through point $(0,-0.5)$ parallel to $x$-axis is selected. With this selection, joint locations of $J_{2}$ and $J_{3}$ are determined. The unit wrench associated with line $l_{23}$ is:

$\mathbf{w}_{23}=[1,0,0.5]^{T}$..

The associated twist $\mathbf{t}_{23}$ is: 
$\mathbf{t}_{23}=\mathbf{C w}_{23}=[0.42,0.29,-0.18]^{T}$.

The location of $\mathbf{t}_{23}, T_{23}$, is determined to be $(1.6111,-2.3333)$.

The ratio $\gamma_{s}$ in Eq. (58) is calculated to be:

$\gamma_{s}=\frac{\left(\mathbf{w}_{12}^{T} \mathbf{t}_{c}\right)\left(\mathbf{w}_{34}^{T} \hat{\mathbf{t}}_{12}\right)\left(\mathbf{w}_{23}^{T} \mathbf{t}_{5}\right)}{\left(\mathbf{w}_{12}^{T} \mathbf{t}_{5}\right)\left(\mathbf{w}_{34}^{T} \mathbf{t}_{5}\right)\left(\mathbf{w}_{23}^{T} \mathbf{t}_{c}\right)}=0.6667$

Since $\gamma_{S}>0$, point $P$ must be outside line segment $J_{5} T_{23}$. Using the set of equations in (59), point $P$ is calculated to be located at $(0.8333,-3.0001)$. The unit twist centered at this location is:

$\mathbf{t}_{p}=[-3.0001,-0.8333,1]^{T}$.

Line $l_{14}$ is uniquely determined by the two points $J_{1}$ and $P$. The unit wrench associated with $l_{14}$ can be determined using Eq. (13):

$\mathbf{w}_{14}=[0.9430,-0.3328,2.5516]^{T}$.

Finally, the intersection of $l_{14}$ and $l_{34}$ identifies the location of $J_{4}$, which is calculated to be $(1,-3.0589)$. The three joint locations $\left(U_{2}, J_{3}, J_{4}\right.$ illustrated in Fig. 12) are:

$\mathbf{r}_{2}=\left[\begin{array}{l}-0.5 \\ -0.5\end{array}\right], \mathbf{r}_{3}=\left[\begin{array}{c}1 \\ -0.5\end{array}\right], \mathbf{r}_{4}=\left[\begin{array}{c}1 \\ -3.0589\end{array}\right]$

Since $T_{12}$ is inside triangle $J_{3} J_{4} J_{5}$, and $T_{34}$ is outside triangle $J_{1} J_{2} J_{5}$ and is inside the area bounded by lines $I_{15}$ and $I_{25}$ (shown in Fig. 12), condition (ii) of Proposition 3 is satisfied. Thus, all five joint compliances for this configuration are positive. The values of the joint compliances are obtained using Eq. (25):

$c_{1}=0.0022 \mathrm{~m} / \mathrm{N}, c_{2}=0.0089 \mathrm{~m} / \mathrm{N}, c_{3}=0.1667 \mathrm{~m} / \mathrm{N}, c_{4}=0.0221 \mathrm{~m} / \mathrm{N}, c_{5}=0.0800 \mathrm{~m} / \mathrm{N}$.

With this synthesis procedure, the serial mechanism configuration with specified locations of joints $J_{1}$ and $J_{5}$ (illustrated in Fig. 12) and the values of all 5 joint compliances are identified. The five joint twists at the configuration are:

$\mathbf{t}_{1}=\left[\begin{array}{c}-2 \\ 2 \\ 1\end{array}\right], \mathbf{t}_{2}=\left[\begin{array}{c}-0.5 \\ 0.5 \\ 1\end{array}\right], \mathbf{t}_{3}=\left[\begin{array}{c}-0.5 \\ -1 \\ 1\end{array}\right], \mathbf{t}_{4}=\left[\begin{array}{c}-3.0589 \\ -1 \\ 1\end{array}\right], \mathbf{t}_{5}=\left[\begin{array}{c}-2 \\ -2 \\ 1\end{array}\right]$.

The result is verified by adding the joint compliance components using Eq. (14):

$\mathbf{C}_{S}=c_{1} \mathbf{t}_{1} \mathbf{t}_{1}^{T}+c_{2} \mathbf{t}_{2} \mathbf{t}_{2}^{T}+c_{3} \mathbf{t}_{3} \mathbf{t}_{3}^{T}+c_{4} \mathbf{t}_{4} \mathbf{t}_{4}^{T}+c_{5} \mathbf{t}_{5} \mathbf{t}_{5}^{T}=\left[\begin{array}{ccc}0.58 \mathrm{~m} / \mathrm{N} & 0.46 \mathrm{~m} / \mathrm{N} & -0.32 \mathrm{~N}^{-1} \\ 0.46 \mathrm{~m} / \mathrm{N} & 0.52 \mathrm{~m} / \mathrm{N} & -0.34 \mathrm{~N}^{-1} \\ -0.32 \mathrm{~N}^{-1} & -0.34 \mathrm{~N}^{-1} & 0.28(\mathrm{~N} \cdot \mathrm{m})^{-1}\end{array}\right]$.

It can be seen that the compliance matrix $\mathbf{C}_{S}$ obtained for the synthesized serial mechanism is the desired compliance matrix C in Eq. (67).

Note that with this procedure, the serial mechanism configuration (with $J_{1}$ and $J_{5}$ specified) is determined by choosing the locations of the other three joints. In construction, the joint connecting sequence does not affect the compliant behavior realized by the serial mechanism, i.e., the same compliance is achieved with joint sequence of $J_{1} J_{4} J_{2} J_{3} J_{5}$ or $J_{1} J_{2} J_{4} J_{3} J_{5}$. 


\subsection{Synthesis with a 5-spring parallel mechanism}

Following the synthesis procedure presented in Section 5.2 , the given stiffness is achieved by a 5-spring parallel mechanism. The component geometry selections used in the process are illustrated in Fig. 13.

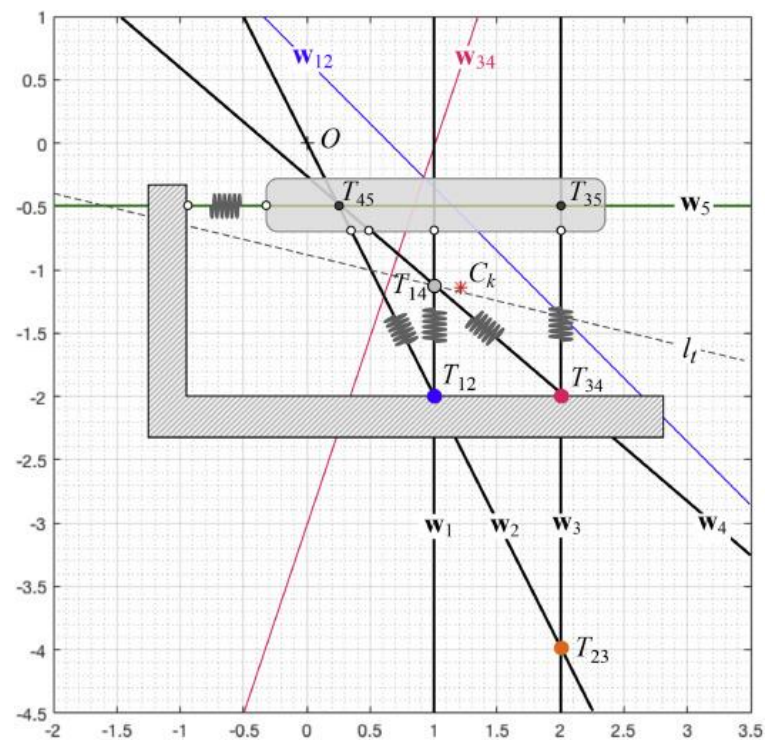

Fig. 13. Stiffness synthesis with a 5-spring mechanism. The desired stiffness is realized by choosing the axes of five springs and their corresponding spring rates in the parallel mechanism.

First, point $T_{12}$ can be chosen arbitrarily. Two spring axes $\left(\mathbf{w}_{1}\right.$ and $\left.\mathbf{w}_{2}\right)$ will meet at this point. In this example, $T_{12}$ is selected to be $(1,-2)$. The unit twist at $T_{12}$ is:

$\mathbf{t}_{12}=[-2,-1,1]^{T}$..

The associated wrench $\mathbf{w}_{12}$ is:

$\mathbf{w}_{12}=\mathbf{K t}_{12}=[-6,6,4]^{T}$.

Normalizing $\mathbf{w}_{12}$ yields the corresponding unit wrench:

$\widehat{\mathbf{w}}_{12}=\frac{\sqrt{2}}{2}\left[-1,1, \frac{2}{3}\right]^{T}$.

The wrench axis of $\mathbf{w}_{12}$ is interpreted geometrically by Eq. (4) and is illustrated in Fig. 13.

The second point $T_{34}$ (where two additional spring axes $\mathbf{w}_{3}$ and $\mathbf{w}_{4}$ will meet) can also be chosen arbitrarily. In this example, $T_{34}$ is chosen to be $(2,-2)$. Using Eq. (3), the unit twist centered at $T_{34}$ is:

$\mathbf{t}_{34}=[-2,-2,1]^{T}$,

and the associated wrench is:

$\mathbf{w}_{34}=\mathbf{K t}_{34}=[-2,-6,-6]^{T}$.

The wrench axis of $\mathbf{w}_{34}$ is shown in Fig. 13.

Next, line $l_{5}$ is selected. To satisfy condition (ii) of Proposition $4, T_{12}$ and $T_{34}$ must be on the same side of $l_{5}$. Also, since $T_{12}$ and $T_{34}$ are located below the stiffness center $C_{k}, \mathbf{w}_{5}$ should be located above $C_{k}$. Here, $l_{5}$ is chosen to pass through point $(0,-0.5)$ and parallel to the $x$-axis. The unit spring wrench $\mathbf{w}_{5}$ associated with this line is: 
$\mathbf{w}_{5}=[1,0,0.5]^{T}$.

Next, choose point $T_{23}$ to determine the spring wrenches $\mathbf{w}_{2}$ and $\mathbf{w}_{3}$. Here, to reduce the range spanned by the springs, point $(2,-4)$ is selected so that $\mathbf{w}_{3}$ is vertical (parallel to the $y$-axis). With this selection, the realization condition of Proposition 4 (ii) is satisfied. The unit twist at $T_{23}$ is calculated to be:

$\mathbf{t}_{23}=[-4,-2,1]^{T}$,

and the corresponding wrench is calculated to be:

$\mathbf{w}_{23}=\mathbf{K t}_{23}=[-14,2,-10]^{T}$.

The distance ratio in Eq. (64) corresponding to the selections of $T_{12}, T_{34}, T_{23}$ and $l_{5}$ is calculated to be:

$\gamma_{p}=\frac{\left(\mathbf{t}_{34}^{T} \mathbf{w}_{12}\right)\left(\mathbf{t}_{c}^{T} \mathbf{w}_{23}\right)\left(\mathbf{t}_{23}^{T} \mathbf{w}_{5}\right)}{\left(\mathbf{t}_{c}^{T} \mathbf{w}_{12}\right)\left(\mathbf{t}_{12}^{T} \mathbf{w}_{5}\right)\left(\mathbf{t}_{34}^{T} \mathbf{w}_{5}\right)}=-6.2222$

Using Eq. (66), the equation for line $I_{t}$ is obtained:

(68) $y=-0.2571 x-0.8857$.

The final point $T_{14}$ must be on line $I_{t}$. Here, $T_{14}$ is selected so that $\mathbf{w}_{1}$ is also vertical. The location of $T_{14}$ can be obtained by solving Eq. (68) with $x=1$. The location of the point is determined to be at $(1,-1.1428)$. The unit twist at this point is:

$\mathbf{t}_{14}=[-1.1428,-1,1]^{T}$.

The axes of the remaining 4 springs are identified by lines $T_{12} T_{14}, T_{12} T_{23}, T_{23} T_{34}$, and $T_{14} T_{34}$ as illustrated in Fig. 13. The 5 spring wrenches in the mechanism are:

$\mathbf{w}_{1}=\left[\begin{array}{l}0 \\ 1 \\ 1\end{array}\right], \mathbf{w}_{2}=\left[\begin{array}{c}0.4472 \\ -0.8944 \\ 0\end{array}\right], \mathbf{w}_{3}=\left[\begin{array}{l}0 \\ 1 \\ 2\end{array}\right], \mathbf{w}_{4}=\left[\begin{array}{c}0.7592 \\ -0.6508 \\ 0.2186\end{array}\right], \mathbf{w}_{5}=\left[\begin{array}{c}1 \\ 0 \\ 0.5\end{array}\right]$.

It can be seen that, for $\left(\mathbf{w}_{1}, \mathbf{w}_{2}\right)$ and $\left(\mathbf{w}_{3}, \mathbf{w}_{4}\right)$, the realization condition in Proposition 4 (ii) is satisfied. Thus all spring stiffnesses must be positive. Since twists $\mathbf{t}_{12}, \mathbf{t}_{23}, \mathbf{t}_{14}$ and $\mathbf{t}_{34}$ are already known, to determine the values of the spring stiffnesses using Eq. (26), only $\mathbf{t}_{35}$ and $\mathbf{t}_{45}$ are needed. It is easy to determine that $T_{35}$ is located at $(2,-0.5)$, , and $T_{45}$ is located at $(0.25,-0.5)$. The two unit twists needed are:

$\mathbf{t}_{35}=[-0.5,-2,1]^{T}, \mathbf{t}_{45}=[-0.5,-0.25,1]^{T}$.

Using these twists, the spring stiffnesses calculated using Eq. (26) are:

$k_{1}=4.6677 \mathrm{~N} / \mathrm{m}, k_{2}=1.6672 \mathrm{~N} / \mathrm{m}, k_{3}=3.1427 \mathrm{~N} / \mathrm{m}, k_{4}=6.7460 \mathrm{~N} / \mathrm{m}, k_{5}=1.7778 \mathrm{~N} / \mathrm{m}$.

With this synthesis procedure, the five spring axes (shown in Fig. 13) and the associated spring constants are identified. The realization of the desired stiffness with the 5 -spring mechanism is verified using Eq. (50):

$\mathbf{K}_{P}=k_{1} \mathbf{w}_{1} \mathbf{w}_{1}^{T}+k_{2} \mathbf{w}_{2} \mathbf{w}_{2}^{T}+k_{3} \mathbf{w}_{3} \mathbf{w}_{3}^{T}+k_{4} \mathbf{w}_{4} \mathbf{w}_{4}^{T}+k_{5} \mathbf{w}_{5} \mathbf{w}_{5}^{T}=\left[\begin{array}{ccc}6 \mathrm{~N} / \mathrm{m} & -4 \mathrm{~N} / \mathrm{m} & 2 \mathrm{~N} \\ -4 \mathrm{~N} / \mathrm{m} & 12 \mathrm{~N} / \mathrm{m} & 10 \mathrm{~N} \\ 2 \mathrm{~N} & 10 \mathrm{~N} & 18 \mathrm{~N} \cdot \mathrm{m}\end{array}\right]$.

It can be seen that the stiffness matrix $\mathbf{K}_{P}$ obtained for the synthesized parallel mechanism is the desired stiffness matrix $\mathbf{K}$ in Eq. (67).

Note that the procedure only identifies the axis of each spring. In parallel mechanism construction, a line spring can be placed at any location along its axis. Also note that, since $\mathbf{w}_{1}$ and $\mathbf{w}_{3}$ are selected to be parallel in this 
example, $\mathbf{t}_{13}$ calculated in Eq. (22) has infinite pitch (centered at infinity). Thus, in calculating spring constants $k_{2}, k_{4}$ and $k_{5}$ using Eq. (26), permutations not containing $\mathbf{t}_{13}$ should be used.

\section{Summary}

In this paper, a geometric approach to passive realization of any given planar compliance with a simple 5-joint serial or 5-spring parallel mechanism is presented. Realization conditions for a 5-component redundant mechanism to achieve an arbitrarily specified planar compliance are presented. Since these conditions impose requirements on the mechanism configuration, they can be geometrically interpreted and illustrated on the plane. Geometry based synthesis procedures to realize a compliance are developed for both types of mechanisms. For serial mechanism synthesis, the procedure identifies the five joint locations and the corresponding joint compliances for the compliance realization. A 5-joint serial mechanism can be constructed based on the component geometry and joint elastic property. For parallel mechanism synthesis, the procedure identifies the five spring axes (lines of action) and the corresponding spring rates for the stiffness realization. A 5-spring parallel mechanism can be constructed based on the geometric and elastic properties of each component. Unlike most of the previous realization approaches, the procedures allow one to choose each component, based on its geometry, from the space of admissible options. Because the space of admissible options is restricted by inequalities, the realization of a compliant behavior with constraints on the mechanism configuration can be accomplished. Any given compliance can be achieved with a mechanism having at most two specified components (i.e., two joint locations of a serial mechanism, or two spring axis lines of a parallel mechanism). The selection of the remaining components is restricted in some way. This ability makes 5component redundant compliant mechanisms more practical in robotic applications, especially for tasks when the mechanism geometry and/or workspace are constrained. Since the approach developed in the paper is completely geometry based, graphic tools can be used to better design an elastic mechanism.

\section{Acknowledgement}

The authors gratefully acknowledge the support of the National Science Foundation under Grant IIS-1427329.

\section{References}

[1]D.E. Whitney, Quasi-static assembly of compliantly supported rigid parts. ASME J. Dyn. Syst. Measure. Control, 104 (1) (1982), pp. 65-77

[2] N. Hogan, Impedance control: an approach to manipulation. ASME J. Dyn. Syst. Measure. Control, 107 (1) (1985), pp. 1-7

[3] R.V. Ham, T.G. Sugar, B. Vanderborght, K.W. Hollander, D. Lefeber, Compliant actuator designs: review of actuators with passive adjustable compliance/controllable stiffness for robotic applications. IEEE Rob. Autom. Mag., 16 (3) (2009), pp. 81-94

[4] S. Huang, J.M. Schimmels, Realization of point planar elastic behaviors using revolute joint serial mechanisms having specified link lengths, Mech. Mach. Theory, 103 (2016), pp. 1-20

[5]S. Huang, J.M. Schimmels, Synthesis of point planar elastic behaviors using 3-joint serial mechanisms of specified construction. ASME J. Mech. Robot., 9 (2017), pp. 011005(1-11)

[6]S. Huang, J.M. Schimmels, Geometric construction-based realization of planar elastic behaviors with parallel and serial manipulators. ASME J. Mech. Robot., 9 (2017), pp. 051006(1-10)

[7] R.S. Ball, A Treatise on the Theory of Screws. London, U.K.: Cambridge University Press (1900)

[8] F.M. Dimentberg, The Screw Calculus and its Applications in Mechanics. Foreign Technology Division, Wright-Patterson Air Force Base, Dayton, Ohio (1965). Document No. FTD-HT-23-1632-67

[9] M. Griffis, J. Duffy, Kinestatic control: a novel theory for simultaneously regulating force and displacement. ASME J. Mech. Des., 113 (4) (1991), pp. 508-515

[10] T. Patterson, H. Lipkin, Structure of robot compliance. ASME J. Mech. Des., 115 (3) (1993), pp. 576-580 
[11] S. Huang, J.M. Schimmels, The Eigenscrew decomposition of spatial stiffness matrices. IEEE Trans. Robot. Autom., 16 (2) (2000), pp. 146-156

[12] J. Loncaric, Normal forms of stiffness and compliance matrices, IEEE J. Robot. Autom., 3 (6) (1987), pp. 567572

[13]S. Huang, J.M. Schimmels, The bounds and realization of spatial stiffnesses achieved with simple springs connected in parallel. IEEE Trans. Robot. Autom., 14 (3) (1998), pp. 466-475

[14] S. Huang, J.M. Schimmels, The bounds and realization of spatial compliances achieved with simple serial elastic mechanisms. IEEE Trans. Robot. Autom., 16 (1) (2000), pp. 99-103

[15] R.G. Roberts, Minimal realization of a spatial stiffness matrix with simple springs connected in parallel. IEEE Trans. Robot. Autom., 15 (5) (1999), pp. 953-958

[16] N. Ciblak, H. Lipkin, Synthesis of cartesian stiffness for robotic applications. Proceedings of the IEEE International Conference on Robotics and Automation, Detroit, MI (1999), pp. 2147-2152

[17] S. Huang, J.M. Schimmels, Achieving an arbitrary spatial stiffness with springs connected in parallel. ASME J. Mech. Des., 120 (4) (1998), pp. 520-526

[18] S. Huang, J.M. Schimmels, The duality in spatial stiffness and compliance as realized in parallel and serial elastic mechanisms. ASME J. Dyn. Syst, Measure. Control, 124 (1) (2002), pp. 76-84

[19] S. Huang, J.M. Schimmels, A classification of spatial stiffness based on the degree of translationalrotational coupling. ASME J. Mech. Des., 123 (3) (2001), pp. 353-358

[20] R.G. Roberts, Minimal realization of an arbitrary spatial stiffness matrix with a parallel connection of simple springs and complex springs. IEEE Trans. Robot. Autom., 16 (5) (2000), pp. 603-608

[21] S. Huang, J.M. Schimmels, Minimal realizations of spatial stiffnesses with parallel or serial mechanisms having concurrent axes. J. Robot. Syst., 18 (3) (2001), pp. 135-246

[22] S. Huang, J.M. Schimmels, Realization of those elastic behaviors that have compliant axes in compact elastic mechanisms. J. Robot. Syst., 19 (3) (2002), pp. 143-154

[23] K. Choi, S. Jiang, Z. Li, Spatial stiffness realization with parallel springs using geometric parameters. IEEE Trans. Robot. Autom., 18 (3) (2002), pp. 264-284

[24]M.B. Hong, Y.J. Choi, Screw system approach to physical realization of stiffness matrix with arbitrary rank. ASME J. Mech. Robot., 1 (2) (2009), pp. 021007(1-8)

[25] S. Huang, J.M. Schimmels, Geometric construction-based realization of spatial elastic behaviors in parallel and serial manipulators. IEEE Trans. Rob., 34 (3) (2018), pp. 764-780

[26] F.P. Petit. Analysis and control of variable stiffness robots, ETH Zurich, Zurich, Switzerland (2014). Ph.D. thesis

[27] M. Verotti, N.P. Belfiore, Isotropic compliance in E(3): feasibility and workspace mapping. ASME J. Mech. Robot., 8 (6) (2016), pp. 061005(1-9)

[28] M. Verotti, P. Masarati, M. Morandini, N. Belfiore, Isotropic compliance in the special euclidean group SE(3). Mech. Mach. Theory, 98 (2016), pp. 263-281

[29]S. Huang, J.M. Schimmels, Realization of an arbitrary planar stiffness with a simple symmetric parallel mechanism. ASME J. Mech. Robot., 3 (4) (2011), pp. 041006(1-8)

[30] S. Huang, J.M. Schimmels, Geometric approach to the realization of planar elastic behaviors with mechanisms having four elastic components. ASME J. Mech. Robot., 10 (2018), pp. 041004(1-13) 